\title{
A geometric Jacquet-Langlands correspondence for $U(2)$ Shimura varieties
}

\author{
David Helm *
}

November 4, 2018

Let $G$ be a unitary group over $\mathbb{Q}$, associated to a CM-field $F$ with totally real part $F^{+}$, with signature $(1,1)$ at all the archimedean places of $F^{+}$. Under certain hypotheses on $F^{+}$, we show that Jacquet-Langlands correspondences between certain automorphic representations of $G$ and representations of a group $G^{\prime}$ isomorphic to $G$ except at infinity can be realized in the cohomology of Shimura varieties attached to $G$ and $G^{\prime}$.

We obtain these Jacquet-Langlands correspondences by studying the bad reduction of a Shimura variety $X$ attached to $G$ at a prime $p$ for which $X$ has " $\Gamma_{0}(p)$ " level structure, and construct a "Deligne-Rapoport" model for $X$. The irreducible components of the special fiber of this model have a global structure that can be explicitly described in terms of Shimura varieties $X^{\prime}$ for unitary groups $G^{\prime}$ isomorphic to $G$ away from infinity.

The weight spectral sequence of Rapoport-Zink then yields an expression for certain pieces of the weight filtration on the étale cohomology of $X$ in terms of the cohomology of $X^{\prime}$. This identifies a piece of this weight filtration with a space of algebraic modular forms for $G^{\prime}$. This result implies certain cases of the Jacquet-Langlands correspondence for $G$ and $G^{\prime}$ in terms of a canonical map between spaces of arithmetic interest, rather than simply as an abstract bijection between isomorphism classes of representations.

2000 MSC Classification: 11G18; 11F55

\section{Introduction}

Let $G$ and $G^{\prime}$ be algebraic groups over $\mathbb{Q}$, isomorphic over all non-archimedean primes of $\mathbb{Q}$ but not necessarily isomorphic over $\mathbb{R}$. In many cases, the JacquetLanglands correspondence predicts an bijection between automorphic representations of $G$ and $G^{\prime}$ satisfying certain technical conditions. (See, for instance, $\mathrm{JL}$ for the case of quaternionic algebraic groups.)

Traditionally, Jacquet-Langlands correspondences for various $G$ and $G^{\prime}$ are established via trace calculations in the theory of automorphic representations.

*University of Texas at Austin; dhelm@math.utexas.edu 
One uses these calculations to show that certain spaces of representations are abstractly isomorphic, and deduces a Jacquet-Langlands correspondence from the existence of such an isomorphism.

In the case of $\mathrm{GL}_{2}$, an observation of Serre $\mathrm{Se}$ shows an alternative way of proceeding: Serre considers spaces of modular forms as sections of line bundles on a modular curve. By restricting these line bundles to the supersingular locus of the characteristic $p$ fiber of such a curve, Serre relates spaces of modular forms mod $p$ to spaces of functions on this supersingular locus. This supersingular locus is a double coset space for a quaternion algebra $B$, and one can interpret the space of functions on such a space as a space of "mod $p$ modular forms" for $B^{\times}$.

In contrast to the traditional approach, which yields only a bijection between isomorphism classes of representations, Serre's result gives a canonical isomorphism between spaces that arise naturally from the geometry of the Shimura varieties attached to the groups $\mathrm{GL}_{2}$ and $B^{\times}$.

More recently, Ghitza [Gh1, Gh2] has generalized Serre's techniques to the case of Siegel modular varieties; he connects automorphic forms for $\mathrm{Sp}_{2 g}$ to algebraic modular forms Gross on a quaternion unitary group. As his results (like those of Serre) use mod $p$ coefficients rather than complex coefficients, his work has consequences for congruences of Siegel modular forms; in particular he can show that all Siegel Hecke eigensystems are cuspidal mod $p$.

One disadvantage of this approach is that it only establishes a correspondence in characteristic $p$. An alternative approach, that also establishes JacquetLanglands correspondences via the geometry of Shimura varieties, is that of Ribet Ri1]. Ribet considers character groups of tori attached to the bad reduction of modular curves and Shimura curves at various primes; these are free $\mathbb{Z}$-modules with actions of Hecke operators for $\mathrm{GL}_{2}$ or a quaternion algebra $B$. He then gives an exact sequence relating these character groups; this amounts to a Jacquet-Langlands correspondence between $\mathrm{GL}_{2}$ and $B^{\times}$with integer coefficients. As with the approach of Serre and Ghizta, Ribet's construction is canonical and contains data about congruences (in fact, it is a key ingredient of his proof of level-lowering.)

Here we obtain similar results in the case of a Shimura variety $X$ attached to a unitary group $G$ whose real points are (up to a factor of $\mathbb{R}^{\times}$) are isomorphic to a product of $U(1,1)$ 's. Such $G$ are defined in terms of a CM field $F$, with totally real part $F^{+}$. Our approach most closely mirrors that of Ribet, in that it makes use of semistable models of these varieties at primes of bad reduction in order to deduce results that hold in characteristic zero (as opposed to the mod $p$ results of Serre and Ghitza, which hold for primes of good reduction.) The reduction theory of Jacobians that plays a prominent role in Ribet's theory is replaced with the theory of vanishing cycles and weight filtrations for these more general Shimura varieties.

Making this argument work requires a through understanding of an analogue of the "Deligne-Rapoport model" for the Shimura varieties under consideration. The local structure of such models is by now very well-understood [RZ1]. Unfortunately, cohomological arguments of the sort we have in mind seem to require 
an understanding of the global structure of various strata in such a model; questions of this nature have recieved far less attention.

The bulk of the paper (sections 3 through 5) is devoted to establishing the necessary global results. In section 3 we define an " $a$-number stratification" of the Shimura varieties under consideration at a prime of good reduction, that is exactly analogous to the stratification of Hilbert modular varieties introduced by E. Goren and F. Oort in [GO, ) and use standard techniques to understand the local structure of the strata. In section 4 we study the global structure of some of these strata; surprisingly, many of the strata that arise in this way turn out to be (nearly) $\left(\mathbb{P}^{1}\right)^{r}$-bundles over Shimura varieties attached to other algebraic groups $G^{\prime}$. These groups are isomorphic to $G$ at all finite places but not at infinity. This is already highly suggestive of a "Jacquet-Langlands" correspondence. Section 5 gives a complete, global description of the components of the special fiber of $X$ at certain primes of bad reduction; the result gives each component of the special fiber as (nearly) a $\left(\mathbb{P}^{1}\right)^{r}$-bundle over one of the Shimura varieties attached to the groups $G^{\prime}$ arising in the previous section.

Section 7 uses these calculations to deduce the main arithmetic results of the paper. The key tool is the Rapoport-Zink weight spectral sequence [RZ2, [Sa], which computes the weight filtration on the étale cohomology of an algebraic variety with semistable reduction in terms of the cohomology of strata of the special fiber. We apply this to a semistable resolution of the model for $X$ described in section 5 . The result is Theorem 7.3 which gives an isomorphism between the highest weight quotient of the the middle étale cohomology of $X$ and a space of algebraic modular forms for a group $G^{\prime}$ that is isomorphic to $G$ at all finite places but (unlike $G$ ) is compact at infinity. This isomorphism is Hecke-equivariant, and therefore provides an explicit, canonical geometric realization of Jacquet-Langlands transfers between $G$ and $G^{\prime}$. For surfaces, the isomorphism is even integral; that is, it works with $\mathbb{Z}_{l}$ coefficients as well as $\mathbb{Q}_{l}$ coefficients.

There are some notational conventions we use throughout the paper. First, we often use the same symbol for an isogeny of abelian varieties, and for the map on de Rham or crystalline cohomology it induces. The dual of an abelian variety $A$ will be denoted $A^{\vee}$.

\section{Basic definitions and properties}

We begin with the definition and basic properties of $U(2)$ Shimura varieties.

Fix a totally real field $F^{+}$, of degree $d$ over $\mathbb{Q}$. Let $E$ be an imaginary quadratic extension of $\mathbb{Q}$, of discriminant $N$, and let $x$ be a square root of $D$ in $E$. Let $F$ be the field $E F^{+}$.

Fix a square root $\sqrt{D}$ of $D$ in $\mathbb{C}$. Then any embedding $\tau: F^{+} \rightarrow \mathbb{R}$ induces two embeddings $p_{\tau}, q_{\tau}: F \rightarrow \mathbb{C}$, via

$$
\begin{aligned}
& p_{\tau}(a+b x)=\tau(a)+\tau(b) \sqrt{N} \\
& q_{\tau}(a+b x)=\tau(a)-\tau(b) \sqrt{N} .
\end{aligned}
$$


Fix a two-dimensional $F$-vector space $\mathcal{V}$, equipped with an alternating, nondegenerate pairing $\langle\rangle:, \mathcal{V} \times \mathcal{V} \rightarrow \mathbb{Q}$. We require that

$$
\langle\alpha x, y\rangle=\langle x, \bar{\alpha} y\rangle
$$

for all $\alpha$ in $F$. (Here $\bar{\alpha}$ denotes the complex conjugate of $\alpha$.)

Each embedding $\tau: F^{+} \rightarrow \mathbb{R}$ gives us a complex vector space $\mathcal{V}_{\tau}=\mathcal{V} \otimes_{F^{+}, \tau} \mathbb{R}$. The pairing $\langle$,$\rangle on \mathcal{V}$ induces a pairing $\langle,\rangle_{\tau}$ on $\mathcal{V}_{\tau}$; this pairing is the "imaginary part" of a unique Hermitian pairing $[,]_{\tau}$ on $\mathcal{V}_{\tau}$. We denote the number of 1 's in the signature of $[,]_{\tau}$ by $r_{\tau}(\mathcal{V})$, and the number of -1 's by $s_{\tau}(\mathcal{V})$. If $\mathcal{V}$ is obvious from the context, we will often omit it, and denote $r_{\tau}(\mathcal{V})$ and $s_{\tau}(\mathcal{V})$ by $r_{\tau}$ and $s_{\tau}$.

We fix a $\hat{\mathbb{Z}}$-lattice $\mathcal{T}$ in $\mathcal{V}\left(\mathbb{A}_{\mathbb{Q}}^{f}\right)$, stable under the action of $\mathcal{O}_{F}$, such that the pairing $\langle$,$\rangle induces a \hat{\mathbb{Z}}$-valued pairing on $\mathcal{T}$.

Finally, we choose a prime $p \in \mathbb{Q}$, unramified in $F$, and split in $E$, such that the cokernel of the induced map $\mathcal{T} \rightarrow \operatorname{Hom}(\mathcal{T}, \hat{\mathbb{Z}})$ has order prime to $p$.

Let $k_{0}$ be a finite field of characteristic $p$ large enough to contain all the residue fields of $\mathcal{O}_{F}$ of characteristic $p$. We fix an embedding of $W\left(k_{0}\right)$ into $\mathbb{C}$. Such a choice associates to each $p_{\tau}$ (resp. $q_{\tau}$ ) as above a map $\mathcal{O}_{F} \rightarrow W\left(k_{0}\right)$, and therefore also a map $\mathcal{O}_{F} / p \mathcal{O}_{F} \rightarrow k_{0}$. In an abuse of notation we denote each of these by $p_{\tau}$ (resp. $\left.q_{\tau}\right)$, and rely on the context to make it clear exactly which we mean.

All these choices allow us to define the algebraic group associated to the Shimura varieties of interest to us. Specifically, we let $G$ be the algebraic group over $\mathbb{Q}$ such that for any $\mathbb{Q}$-algebra $R, G(R)$ is the $\operatorname{subgroup~of~} \operatorname{Aut}_{F}\left(\mathcal{V} \otimes_{\mathbb{Q}} R\right)$ consisting of all $g$ such that there exists an $r$ in $R^{\times}$with $\langle g x, g y\rangle=r\langle x, y\rangle$ for all $x$ and $y$ in $\mathcal{V} \otimes_{\mathbb{Q}} R$. In particular, $G(\mathbb{R})$ is the subgroup of

$$
\prod_{\tau: F^{+} \rightarrow \mathbb{R}} \mathrm{GU}\left(r_{\tau}, s_{\tau}\right)
$$

consisting of those tuples $\left(g_{\tau}\right)_{\tau: F^{+} \rightarrow \mathbb{R}}$ such that the "similitude ratio" of $g_{\tau}$ is the same for all $\tau$.

Now fix a compact open subgroup $U$ of $G\left(\mathbb{A}_{\mathbb{Q}}^{f}\right)$, such that $U$ preserves $\mathcal{T}$, and such that $U_{p}$ is the largest subgroup of $G\left(\mathbb{Q}_{p}\right)$ preserving $\mathcal{T}_{p}$. If $U$ is sufficiently small, there exists a scheme $X_{U}(\mathcal{V})$ over $W\left(k_{0}\right)$ representing the functor that takes an $W\left(k_{0}\right)$-scheme $S$ to the set of isomorphism classes of triples $(A, \lambda, \rho)$ where

1. $A$ is an abelian scheme over $S$ of dimension $2 d$, with an action of $\mathcal{O}_{F}$, such that the characteristic polynomial of $\alpha \in \mathcal{O}_{F}$ as an endomorphism of $\operatorname{Lie}(A / S)$ is given by

$$
\prod_{\tau: F^{+} \rightarrow \mathbb{R}}\left(x-p_{\tau}(\alpha)\right)^{r_{\tau}(\mathcal{V})}\left(x-q_{\tau}(\alpha)\right)^{s_{\tau}(\mathcal{V})} .
$$

2. $\lambda$ is a polarization of $A$, of degree prime to $p$, such that the Rosati involution associated to $\lambda$ induces complex conjugation on $\mathcal{O}_{F} \subset \operatorname{End}(A)$. 
3. $\rho$ is a $U$-orbit of isomorphisms $T_{\hat{\mathbb{Z}}(p)} A \rightarrow \mathcal{T}^{(p)}$, sending the Weil pairing on $T_{\hat{\mathbb{Z}}(p)} A$ to a scalar multiple of the pairing $\langle$,$\rangle on \mathcal{T}^{(p)}$. (Here $T_{\hat{\mathbb{Z}}(p)} A$ denotes the product over all $l \neq p$ of the $l$-adic Tate modules of $A$.)

We call a scheme $X_{U}(\mathcal{V})$ arising in this way a $U(2)$ Shimura variety.

Remark 2.1 The condition on the characteristic polynomials of elements of $\mathcal{O}_{F}$ acting on $\operatorname{Lie}(A / S)$ can be reformulated in a more illuminating manner. If $M$ is a locally free sheaf on $S$ with an action of $\mathcal{O}_{F}$, then $M$ admits an action of $\mathcal{O}_{F} \otimes W\left(k_{0}\right)$, and hence breaks up as a direct sum:

$$
M=\bigoplus_{\varphi: \mathcal{O}_{F} \rightarrow W\left(k_{0}\right)} M_{\varphi}
$$

where $M_{\varphi}$ is the locally free submodule of $M$ on which $\mathcal{O}_{F}$ acts via the embedding $\varphi$ of $\mathcal{O}_{F}$ in $W\left(k_{0}\right)$. Our choice of embedding of $W\left(k_{0}\right)$ in $\mathbb{C}$ allows us to consider each complex embedding $p_{\tau}$ or $q_{\tau}$ of $\mathcal{O}_{F}$ as such a character. In this language, the characteristic polynomial condition is equivalent to requiring that $\operatorname{Lie}(A / S)_{p_{\tau}}$ and $\operatorname{Lie}(A / S)_{q_{\tau}}$ are locally free of ranks $r_{\tau}(\mathcal{V})$ and $s_{\tau}(\mathcal{V})$, respectively.

Since we have taken $U_{p}$ to be the subgroup of $G\left(\mathbb{Q}_{p}\right)$ preserving $\mathcal{T}_{p}, U_{p}$ is a hyperspecial subgroup of $G\left(\mathbb{Q}_{p}\right)$. It follows that the $\bmod p$ reduction $X_{U}(\mathcal{V}) / k_{0}$ is a smooth variety. Its dimension is equal to the number of $\tau$ such that $r_{\tau}(\mathcal{V})=$ $s_{\tau}(\mathcal{V})=1$, or equivalently to the sum

$$
\sum_{\tau} r_{\tau}(\mathcal{V}) s_{\tau}(\mathcal{V})
$$

We will also be interested in a variant of $X_{U}(\mathcal{V})$ that has some level structure at $p$. In particular, we define $X_{U, p}(\mathcal{V})$ to be the $W\left(k_{0}\right)$-scheme representing the functor that takes $S$ to the set of isomorphism classes of tuples $\left(A, \lambda, \rho, B, \lambda^{\prime}, \phi\right)$, where

1. $(A, \lambda, \rho)$ is an $S$-point of $X_{U}(\mathcal{V})$.

2. $B$ is an abelian scheme over $S$ of dimension $2 d$, with an action of $\mathcal{O}_{F}$.

3. $\lambda^{\prime}$ is a polarization of $B$, of degree prime to $p$.

4. $\phi: A \rightarrow B$ is an $\mathcal{O}_{F}$-isogeny of degree $p^{2 d}$, satisfying:

- $p \lambda=\phi^{\vee} \lambda^{\prime} \phi$

- for each prime $\mathfrak{p}$ of $\mathcal{O}_{F}$ over $p, \operatorname{ker} \phi \cap A[\mathfrak{p}]$ is a finite flat group scheme over $S$ of order equal to the norm of $\mathfrak{p}$, and

- the cokernel of the map $H_{\mathrm{DR}}^{1}(B / S) \rightarrow H_{\mathrm{DR}}^{1}(A / S)$ is a locally free $\mathcal{O}_{F} \otimes \mathcal{O}_{S} / p$-module of rank one. 
Unlike $X_{U}(\mathcal{V}), X_{U, p}(\mathcal{V})$ is not smooth; in fact, in characteristic $p$ it is not even irreducible. The structure of its irreducible components will be discussed in section 5 ,

Remark 2.2 The schemes $X_{U}(\mathcal{V})$ and $X_{U, p}(\mathcal{V})$ depend not only on $U$ and $\mathcal{V}$ but on all of the choices we have made in this section. To avoid clutter, we have chosen to suppress most of these choices in our notation.

One can also consider a more general version of the above moduli problems. Let $F^{+}, E$, and $F$ be as above, and let $D$ be a central simple algebra over $F$ of dimension 4. Fix an involution $\alpha \mapsto \bar{\alpha}$ of $D$, of the second kind, and a maximal order $\mathcal{O}_{D}$ of $D$ stable under this involution.

Let $\mathcal{V}$ now denote a free left $D$-module of rank one, equipped with a nondegenerate alternating pairing

$$
\langle,\rangle: \mathcal{V} \times \mathcal{V} \rightarrow \mathbb{Q}
$$

with the property that

$$
\langle\alpha x, y\rangle=\langle x, \bar{\alpha} y\rangle
$$

for all $\alpha$ in $D$. For each $\tau: F^{+} \rightarrow \mathbb{R}, \mathcal{V} \otimes_{F^{+}, \tau} \mathbb{R}$ is a free $D \otimes_{F^{+}, \tau} \mathbb{R}$-module of rank one. Since $F$ is purely imaginary, $D \otimes_{F^{+}, \tau} \mathbb{R}$ is isomorphic to $G_{2}(\mathbb{C})$, so choosing a suitable idempotent of $e$ of $D \otimes_{F^{+}, \tau} \mathbb{R}$, such that $e=\bar{e}$, we find that $e\left(\mathcal{V} \otimes_{F^{+}, \tau} \mathbb{R}\right)$ is a two-dimensional complex vector space with equipped with a nondegenerate Hermitian pairing induced by $\langle$,$\rangle . We let r_{\tau}(\mathcal{V})$ and $s_{\tau}(\mathcal{V})$ be the number of 1 's and -1 's, respectively, in the signature of this pairing.

Finally, we fix a $\hat{\mathbb{Z}}$-lattice $\mathcal{T}$ in $\mathcal{V}\left(\mathbb{A}_{\mathbb{Q}}^{f}\right)$, stable under the action of $\mathcal{O}$, such that $\langle$,$\rangle induces a \hat{\mathbb{Z}}$-valued pairing on $\mathcal{T}$. We fix a $p$, unramified in $F^{+}$and split in $E$, such that $B$ is split at $p$ and the cokernel of the $\operatorname{map} \mathcal{T} \rightarrow \operatorname{Hom}(\mathcal{T}, \hat{\mathbb{Z}})$ induced by $\langle$,$\rangle has order prime to p$.

Then for $G \subset \operatorname{Aut}_{D}(\mathcal{V})$ the subgroup of automorphisms which take $\langle$,$\rangle to$ a scalar multiple of itself, and $U$ a compact open subgroup of $G$, preserving $\mathcal{T}$ such that $U_{p}$ is the subgroup of $G_{p}$ fixing $\mathcal{T}_{p}$, we can associate a Shimura variety $X_{U}^{D}(\mathcal{V})$. The corresponding moduli problem over $W\left(k_{0}\right)$ parametrizes tuples $(A, \lambda, \rho)$, where

1. $A$ is an abelian scheme over $S$ of dimension $4 d$, with an action of $\mathcal{O}_{D}$, such that the characteristic polynomial of $\alpha \in \mathcal{O}_{F}$ as an endomorphism of $\operatorname{Lie}(A / S)$ is given by

$$
\prod_{\tau: F^{+} \rightarrow \mathbb{R}}\left(x-p_{\tau}(\alpha)\right)^{2 r_{\tau}(\mathcal{V})}\left(x-q_{\tau}(\alpha)\right)^{2 s_{\tau}(\mathcal{V})} .
$$

2. $\lambda$ is a polarization of $A$, of degree prime to $p$, such that the Rosati involution associated to $\lambda$ induces the involution $\alpha \mapsto \bar{\alpha}$ on $\mathcal{O}_{D}$.

3. $\rho$ is a $U$-orbit of isomorphisms $T_{\widehat{\mathbb{Z}}(p)} A \rightarrow \mathcal{T}^{(p)}$, sending the Weil pairing on $T_{\hat{\mathbb{Z}}^{(p)}} A$ to a scalar multiple of the pairing $\langle$,$\rangle on \mathcal{T}^{(p)}$. (Here $T_{\hat{\mathbb{Z}}^{(p)}} A$ denotes the product over all $l \neq p$ of the $l$-adic Tate modules of $A$.) 
Similarly, one defines $X_{U, p}^{D}(\mathcal{V})$ to be the space that parameterizes tuples $\left(A, \lambda, \rho, B, \lambda^{\prime}, \phi\right)$, where

1. $(A, \lambda, \rho)$ is an $S$-point of $X_{U}^{D}(\mathcal{V})$.

2. $B$ is an abelian scheme over $S$ of dimension $4 d$, with an action of $\mathcal{O}_{D}$.

3. $\lambda^{\prime}$ is a polarization of $B$, of degree prime to $p$.

4. $\phi: A \rightarrow B$ is an $\mathcal{O}_{F}$-isogeny of degree $p^{4 d}$, satisfying:

- $p \lambda=\phi^{\vee} \lambda^{\prime} \phi$

- for each prime $\mathfrak{p}$ of $\mathcal{O}_{F}$ over $p, \operatorname{ker} \phi \cap A[\mathfrak{p}]$ is a finite flat group scheme over $S$ of order equal to the norm of $\mathfrak{p}$ squared, and

- the cokernel of the map $H_{\mathrm{DR}}^{1}(B / S) \rightarrow H_{\mathrm{DR}}^{1}(A / S)$ is a locally free $\mathcal{O}_{F} \otimes \mathcal{O}_{S} / p$-module of rank two.

Note that for $D=M_{2}(F)$, with involution equal to conjugate transpose, this is equivalent to our original moduli problem. Specifically, given a point $(A, \lambda, \rho)$ of $X_{U}(\mathcal{V})$, we obtain a point $\left(A^{2}, \lambda^{2}, \rho^{2}\right)$ of $X_{U}^{D}(\mathcal{V})$ by letting $\mathrm{GL}_{2}\left(\mathcal{O}_{F}\right)$ act on $A^{2}$ in the obvious way. Conversely, given a point $(A, \lambda, \rho)$ of $X_{U}^{D}(\mathcal{V})$, for a suitable idempotent $e$ of $M_{2}\left(\mathcal{O}_{F}\right)$ (i.e., one stable under conjugate transpose) $\lambda$ induces a prime-to- $p$ polarization $e \lambda: e A \rightarrow(e A)^{\vee}$, and so $(e A, e \lambda, e \rho)$ is a point of $X_{U}(\mathcal{V})$.

For the purposes of most of this paper, it will matter little whether we work with $X_{U}(\mathcal{V})$ or $X_{U}^{D}(\mathcal{V})$ for some $D$. In particular, all of our constructions are done in terms of the $p$-divisible groups of the moduli objects we consider. Since $D$ is split at $p$, we can fix an idempotent $e$ of $\mathcal{O}_{D} \otimes \mathbb{Z}_{p}$ such that $e=\bar{e}$. Then if we have a point $(A, \lambda, \rho)$ of $X_{U}^{D}(\mathcal{V}), e A\left[p^{\infty}\right]$ behaves exactly like the $p$-divisible group of a moduli object of $X_{U}(\mathcal{V})$.

Therefore, to avoid cluttering our notation with idempotents everywhere, for the bulk of the paper we consider only the schemes $X_{U}(\mathcal{V})$ and $X_{U, p}(\mathcal{V})$. All of our results hold more generally for any $X_{U}^{D}(\mathcal{V})$ for $D$ as above (in particular, split at $p$ ). We leave it to the reader to determine how the proofs given here may be adapted to this case.

\section{A stratification of $U(2)$ Shimura varieties mod $p$}

Fix choices for $F^{+}, E, \mathcal{V}$, and $U$, and let $r_{\tau}=r_{\tau}(\mathcal{V})$ and $s_{\tau}=s_{\tau}(\mathcal{V})$ for all $\tau$. Let $X=X_{U}(\mathcal{V})_{k_{0}}$ be the fiber over $k_{0}$ of the $U(2)$ Shimura variety arising from these choices as in section 2. Our first goal will be to construct a stratification on $X$. We do this by adapting a closely related construction of Goren and Oort [GO] for Hilbert modular varieties. The stratification we obtain is a "generalized Ekedahl-Oort" stratification for $U(2)$ Shimura varieties; these have been studied in considerable generality by many authors; see for instance [O], $\mathrm{Mo}$, or [We]. 
Let $\mathcal{A}$ be the universal abelian variety over $X$. Then the relative Lie algebra $\operatorname{Lie}(\mathcal{A} / X)$ is a locally free $\mathcal{O}_{X}$-module of rank $2 d$, and decomposes as a direct sum:

$$
\operatorname{Lie}(\mathcal{A} / X)=\bigoplus_{\tau \in \mathbb{S}}\left(\operatorname{Lie}(\mathcal{A} / X)_{p_{\tau}} \oplus \operatorname{Lie}(\mathcal{A} / X)_{q_{\tau}}\right),
$$

where $\mathbb{S}=\operatorname{Hom}_{\text {alg }}\left(F^{+}, \mathbb{R}\right)$ as in remark 2.1 Moreover, the bundles $\operatorname{Lie}(\mathcal{A} / X)_{p_{\tau}}$ and $\operatorname{Lie}(\mathcal{A} / X)_{q_{\tau}}$ are locally free of rank $r_{\tau}$ and $s_{\tau}$, respectively.

Fix a $\tau$ such that $r_{\tau}=s_{\tau}=1$. The Verschiebung $V: \mathcal{A}^{(p)} \rightarrow \mathcal{A}$ induces maps

$$
\operatorname{Lie}\left(\mathcal{A}^{(p)} / X\right)_{p_{\tau}} \rightarrow \operatorname{Lie}(\mathcal{A} / X)_{p_{\tau}}
$$

for all $\tau$. Moreover, there is a natural isomorphism:

$$
\operatorname{Lie}\left(\mathcal{A}^{(p)} / X\right) \cong F_{\text {abs }}^{*}(\operatorname{Lie}(\mathcal{A} / X)),
$$

where $F_{\text {abs }}$ is the absolute Frobenius on $X$. Thus we have a natural isomorphism:

$$
\operatorname{Lie}\left(\mathcal{A}^{(p)} / X\right)_{p_{\tau}} \cong\left(F_{\text {abs }}^{*}(\operatorname{Lie}(\mathcal{A} / X))\right)_{p_{\tau}} .
$$

Let $\sigma: k_{0} \rightarrow k_{0}$ be the Frobenius automorphism of $k_{0}$. Via our choice of embedding of $W\left(k_{0}\right)$ in $\mathbb{C}$ in section 2, an embedding $\tau: F^{+} \rightarrow \mathbb{R}$ corresponds to a unique map $\mathcal{O}_{F^{+}} \rightarrow k_{0}$. Composing this map with $\sigma$, we obtain a new map $\mathcal{O}_{F^{+}} \rightarrow k_{0}$. We let $\sigma \tau$ denote the map $F^{+} \rightarrow \mathbb{R}$ corresponding to this new map. Since the absolute Frobenius induces $\sigma$ on $k_{0}$, we have

$$
\left(F_{\text {abs }}^{*}(\operatorname{Lie}(\mathcal{A} / X))\right)_{p_{\tau}} \cong F_{\text {abs }}^{*}\left(\operatorname{Lie}(\mathcal{A} / X)_{p_{\sigma \tau}}\right)
$$

with this convention.

Suppose further that $r_{\sigma \tau}=s_{\sigma \tau}=1$, so that $\operatorname{Lie}(\mathcal{A} / X)_{p_{\sigma \tau}}$ is a line bundle. Pullback of a line bundle by Frobenius sends transition functions to their $p$ th powers, and therefore sends a line bundle to its $p$ th tensor power. We thus obtain a canonical isomorphism

$$
\operatorname{Lie}\left(\mathcal{A}^{(p)} / X\right)_{p_{\tau}} \cong \operatorname{Lie}(\mathcal{A} / X)_{p_{\sigma \tau}}^{\otimes p} .
$$

In particular, for each $\tau$, Verschiebung yields a section $h_{\tau}$ of the line bundle $\operatorname{Hom}\left(\operatorname{Lie}(\mathcal{A} / X)_{p_{\sigma \tau}}^{\otimes p}, \operatorname{Lie}(\mathcal{A} / X)_{p_{\tau}}\right)$.

Remark 3.1 These $h_{\tau}$ are close analogues of the "partial Hasse invariants" constructed by Goren in the setting of Hilbert modular varieties Go.

Remark 3.2 We could just as easily have done this construction with the $q_{\tau}$ instead of with the $p_{\tau}$. The existence of a prime-to- $p$ polarization on $\mathcal{A}$ means that the $p_{\tau}$ and $q_{\tau}$ components of $\operatorname{Lie}(\mathcal{A} / X)$ are dual to each other, and hence that the bundle and section obtained from considering the $q_{\tau}$ components are canonically isomorphic to those obtained from the $p_{\tau}$ components.

Proposition 3.3 Let $k$ be a perfect field of characteristic $p, x \in X(k)$, and $A / k$ the abelian variety corresponding to $x$. Then $x$ lies in $Z\left(h_{\tau}\right)$ if and only if the $k$-vector space $\operatorname{Hom}\left(\alpha_{p}, A[p]\right)_{p_{\tau}}$ has dimension at least one. 
Proof. Let $\mathcal{D}$ denote the contravariant Dieudonné module of $A[p]$. The action of $\mathcal{O}_{F}$ on $\mathcal{D}$ decomposes $\mathcal{D}$ into a direct sum:

$$
\mathcal{D}=\bigoplus_{\tau} \mathcal{D}_{p_{\tau}} \oplus \mathcal{D}_{q_{\tau}} .
$$

Let $F_{\mathcal{D}}$ and $V_{\mathcal{D}}$ denote the Frobenius and Verschiebung endomorphisms of $\mathcal{D}$. Then $\operatorname{Hom}\left(\alpha_{p}, A[p]\right)_{p_{\tau}}$ is nonzero if and only if the quotient

$$
\left(\mathcal{D} /\left(\operatorname{im} F_{\mathcal{D}}+\operatorname{im} V_{\mathcal{D}}\right)\right)_{p_{\tau}}
$$

is nonzero. This latter module can also be written as

$$
\mathcal{D}_{p_{\tau}} /\left(F_{\mathcal{D}}\left(\mathcal{D}_{p_{\sigma^{-1} \tau}}\right)+V_{\mathcal{D}}\left(\mathcal{D}_{p_{\sigma \tau}}\right)\right) .
$$

On the other hand, one has a canonical isomorphism

$$
\mathcal{D} \cong H_{\mathrm{DR}}^{1}(A / k),
$$

that sends $V_{\mathcal{D}}(\mathcal{D})$ to the subspace $\operatorname{Lie}(A / k)^{*}$ of $H_{\mathrm{DR}}^{1}(A / k)$. In particular, $V_{\mathcal{D}}\left(\mathcal{D}_{p_{\tau}}\right)$ has dimension $r_{\sigma^{-1} \tau}=1$. Since the image of $V_{\mathcal{D}}$ is equal to the kernel of $F_{\mathcal{D}}$, we also have that $F_{\mathcal{D}}\left(\mathcal{D}_{p_{\sigma_{-1}}}\right)$ is one dimensional. It follows that $\operatorname{Hom}\left(\alpha_{p}, A[p]\right)_{p_{\tau}}$ is nonempty if and only if $V_{\mathcal{D}}\left(\mathcal{D}_{p_{\sigma \tau}}\right)$ equals $F_{\mathcal{D}}\left(\mathcal{D}_{p_{\sigma-1}{ }_{\tau}}\right)$.

In terms of de Rham cohomology, this means that $\operatorname{Hom}\left(\alpha_{p}, A[p]\right)_{p_{\tau}}$ is nonzero if and only if $\operatorname{Lie}(A / k)_{p_{\tau}}^{*}$ is contained in $\operatorname{Fr}\left(H_{\mathrm{DR}}^{1}\left(A^{(p)} / k\right)\right)$, where $\operatorname{Fr}$ is the relative Frobenius $A \rightarrow A^{(p)}$. The latter is the kernel of the map induced by Verschiebung on de Rham cohomology. Thus $\operatorname{Hom}\left(\alpha_{p}, A[p]\right)_{p_{\tau}}$ is nonzero if and only if the map

$$
h_{\tau}^{*}: \operatorname{Lie}(A)_{p_{\tau}}^{*} \rightarrow \operatorname{Lie}\left(A^{(p)}\right)_{p_{\tau}}^{*}
$$

induced by Verschiebung is the zero map, as required.

For $S$ a subset of those $\tau$ with $r_{\tau}=r_{\sigma^{-1} \tau}=1$, let $X_{S}$ denote the (schemetheoretic) intersection of the $Z\left(h_{\tau}\right)$ for $\tau$ in $S$.

Proposition 3.4 If $X_{S} \subset X$ is nonempty, then $X_{S}$ is smooth, of codimension equal to the cardinality of $S$. Moreover, if $S$ is nonempty, then $X_{S}$ is proper.

Before proving this we recall some basic facts about the deformation theory of abelian varieties:

Let $S$ be a scheme, and $S^{\prime}$ a nilpotent thickening of $S$ equipped with divided powers. Let $\mathcal{C}_{S^{\prime}}$ denote the category of abelian varieties over $S^{\prime}$, and $\mathcal{C}_{S}$ denote the category of abelian varieties over $S$. For $A$ an object of $\mathcal{C}_{S^{\prime}}$, let $\bar{A}$ denote its base change to $\mathcal{C}_{S}$.

Fix an $A$ in $\mathcal{C}_{S^{\prime}}$, and consider the module $H_{\text {cris }}^{1}(\bar{A} / S)_{S^{\prime}}$. This is a locally free $\mathcal{O}_{S^{\prime}}$-module, and we have a canonical isomorphism:

$$
H_{\text {cris }}^{1}(\bar{A} / S)_{S^{\prime}} \cong H_{\mathrm{DR}}^{1}\left(A / S^{\prime}\right) .
$$

Moreover, we have a natural subbundle

$$
\operatorname{Lie}\left(A / S^{\prime}\right)^{*} \subset H_{\mathrm{DR}}^{1}\left(A / S^{\prime}\right) .
$$


This gives us a subbundle of $H_{\text {cris }}^{1}(\bar{A} / S)_{S^{\prime}}$ that lifts the subbundle Lie $(\bar{A} / S)^{*}$ of $H_{\mathrm{DR}}^{1}(\bar{A} / S)$.

In fact, knowing this lift allows us to recover $A$ from $\bar{A}$. More precisely, let $\mathcal{C}_{S}^{+}$denote the category of pairs $(\bar{A}, \omega)$, where $\bar{A}$ is an object of $\mathcal{C}_{S}$ and $\omega$ is a subbundle of $H_{\text {cris }}^{1}(\bar{A} / S)_{S^{\prime}}$ that lifts $\operatorname{Lie}(\bar{A} / S)^{*}$. Then the construction outlined above gives us a functor from $\mathcal{C}_{S^{\prime}}$ to $\mathcal{C}_{S}^{+}$.

Theorem 3.5 (Grothendieck) The functor $\mathcal{C}_{S^{\prime}} \rightarrow \mathcal{C}_{S}^{+}$defined above is an equivalence of categories.

Proof. For an outline of the basic ideas, see [Gr], pp. 116-118. A complete proof can be found in $[\mathrm{MM}$, primarily chapter II, section 1 .

We are now in a position to prove Proposition 3.4 First note that $X_{S}$ is defined by the vanishing of sections of $n$ line bundles, where $n$ is the cardinality of $S$. Thus $X_{S}$ has codimension at most $n$ in $X$. It suffices to show that the tangent space to $X_{S}$ at any $k$-point $x=(A, \lambda, \rho)$ of $X_{S}$ ( $k$ finite) has codimension $n$ in the tangent space to $X$.

Let $\mathbb{I}$ be the scheme Spec $k[\epsilon] / \epsilon^{2}$. The first-order deformations of $A$ are in bijection with the lifts $\omega$ of $\operatorname{Lie}(A / k)^{*}$ to a subbundle of $H_{\text {cris }}^{1}(A / k)_{\mathbb{I}}$. The $\mathcal{O}_{F^{-}}$ action on $A$ lifts to the first order deformation given by $\omega$ if and only if $\omega$ is of the form

$$
\omega=\bigoplus_{\tau} \omega_{p_{\tau}} \oplus \omega_{q_{\tau}},
$$

where $\omega_{p_{\tau}}\left(\right.$ resp. $\left.\omega_{q_{\tau}}\right)$ is a lift of $\operatorname{Lie}(A / k)_{p_{\tau}}^{*}\left(\operatorname{resp} . \operatorname{Lie}(A / k)_{q_{\tau}}^{*}\right)$ to $\left(H_{\text {cris }}^{1}(A / k)_{\mathbb{I}}\right)_{p_{\tau}}$ (resp. $\left(H_{\text {cris }}^{1}(A / k)_{\mathbb{I}}\right)_{q_{\tau}}$.)

The (prime-to- $p$ ) polarization $\lambda$ on $A$ induces a perfect pairing on $H_{\text {cris }}^{1}(A / k)_{\mathbb{I}}$. Since the Rosati involution induces complex conjugation on $\mathcal{O}_{F}$, this restricts to a perfect pairing:

$$
\left(H_{\text {cris }}^{1}(A / k)_{\mathbb{I}}\right)_{p_{\tau}} \times\left(H_{\text {cris }}^{1}(A / k)_{\mathbb{I}}\right)_{q_{\tau}} \rightarrow k[\epsilon] / \epsilon^{2} .
$$

By Theorem 3.5. $\lambda$ lifts to the first-order deformation given by $\omega$ if and only if $\omega_{q_{\tau}}=\omega_{p_{\tau}}^{\perp}$ for all $\tau$, where $\perp$ denotes orthogonal complement with respect to the pairing above. (c.f. [d], Corollary 2.2).

Finally, for any choice of $\omega$ for which the $\mathcal{O}_{F}$-action and $\lambda$ lift to the corresponding deformation, the level structure $\rho$ lifts uniquely. Thus specifying a tangent vector to $X$ at $x$ is equivalent to specifying, for each $\tau$, a lift of the the $r_{\tau}$-dimensional subspace $\operatorname{Lie}(A / k)_{p_{\tau}}$ of the two dimensional vector space $H_{\mathrm{DR}}^{1}(A / k)_{p_{\tau}}$ to a subbundle $\omega_{p_{\tau}}$ of $\left(H_{\mathrm{cris}}^{1}(A / k)_{\mathbb{I}}\right)_{p_{\tau}}$. If $r_{\tau}$ is zero or two, the lift is obviously unique. For each $\tau$ with $r_{\tau}=1$, such lifts form a one-dimensional $k$-vector space.

Let $v$ be a tangent vector to $X$ at $x$, corresponding to a lift $(\hat{A}, \hat{\lambda}, \hat{\rho})$ of $(A, \lambda, \rho)$. For $\tau$ such that $r_{\tau}=r_{\sigma^{-1} \tau}=1$, the vector $v$ lies in $X_{\{\tau\}}$ if and only if the map

$$
V: \operatorname{Lie}(\hat{A} / \mathbb{I})_{p_{\tau}}^{*} \rightarrow \operatorname{Lie}\left(\hat{A}^{(p)} / \mathbb{I}\right)_{p_{\tau}}^{*}
$$


is the zero map. This in turn is true if and only if the lift $\omega_{p_{\tau}}$ coming from $v$ is contained in the kernel of the map

$$
V:\left(H_{\text {cris }}^{1}(A / k)_{\mathbb{I}}\right)_{p_{\tau}} \rightarrow\left(H_{\text {cris }}^{1}\left(A^{(p)} / k\right)_{\mathbb{I}}\right)_{p_{\tau}} .
$$

Since both $\omega_{p_{\tau}}$ and this kernel are subbundles of rank one, this condition determines $\omega_{p_{\tau}}$ uniquely.

Thus to specify a tangent vector to $X_{S}$ at $x$, it suffices to specify $\omega_{p_{\tau}}$ for $\tau$ not in $S$. In particular the tangent space to $X_{S}$ at $x$ has codimension $n$ in the tangent space to $X$ at $x$, as required.

It remains to show that $X_{S}$ is proper for $S$ nonempty. Let $R$ be a discrete valuation ring containing $k_{0}$, let $K$ be its field of fractions, and let $k$ be its residue field. Let $x: \operatorname{Spec} K \rightarrow X_{S}$ be a point of $X_{S}$, and let $A / K$ be the corresponding abelian variety. Then there exists a finite extension $K^{\prime}$ of $K$ such that $A_{K^{\prime}}$ has either good or multiplicative reduction.

Suppose it had multiplicative reduction, and let $\mathbb{T}$ be the maximal subtorus of the reduction. The character group of $\mathbb{T}$ is a free $\mathbb{Z}$-module of rank at most $2 d$ on which $\mathcal{O}_{F}$ acts; this is only possible if $\mathbb{T}$ had rank exactly $2 d$, in which case $A_{K^{\prime}}$ has purely toric reduction. But then Verschiebung would induce an isomorphism on the Lie algebra of the special fiber, which is impossible because it has a kernel on the general fiber.

Thus $A_{K^{\prime}}$ has good reduction. In particular $A_{K^{\prime}}$ extends to an abelian scheme over Spec $R^{\prime}$, where $R^{\prime}$ is the integral closure of $R$ in $K^{\prime}$. The polarization and level structure on $A_{K^{\prime}}$ extend to $R^{\prime}$ as well. We thus obtain a map Spec $R^{\prime} \rightarrow X_{S}$ extending the map $\operatorname{Spec} K^{\prime} \rightarrow X_{S}$, so $X_{S}$ is proper.

Remark 3.6 It follows immediately from this result that the nonempty $X_{S}$ are the closed strata in a stratification (the $a$-number stratification) of $X$.

Lemma 3.7 If $r_{\tau}=s_{\tau}=1$ for all $\tau$, then $X_{S}$ is nonempty for all $S$.

Proof. It suffices to prove this for $S=\mathbb{S}$. Let $\mathcal{E}$ be a supersingular elliptic curve over $\overline{\mathbb{F}}_{p}$, and let $A$ be the abelian variety $\mathcal{E} \otimes_{\mathbb{Z}} \mathcal{O}_{F}$; that is, the abelian variety such that $A(S)=\mathcal{E}(S) \otimes_{\mathbb{Z}} \mathcal{O}_{F}$ for all schemes $S$. Then both $T_{\mathbb{\mathbb { Z }}(p)} A$ and $\mathcal{T}^{(p)}$ are free $\hat{\mathcal{O}}_{F}^{(p)}$-modules of rank two. Fix any isomorphism $\rho$ between them, compatible with the action of $\mathcal{O}_{F}$. The natural polarization on $E$ then induces a polarization on $A$, that identifies $T_{\hat{\mathbb{Z}}^{(p)}} A^{\vee}$ with $\operatorname{Hom}\left(\mathcal{T}^{(p)}, \hat{\mathbb{Z}}^{(p)}\right)$. Thus the pairing on $\mathcal{T}^{(p)}$ determines a polarization $\lambda$ of $A$, of degree prime to $p$. Moreover, we have isomorphisms

$$
\operatorname{Lie}\left(A / \overline{\mathbb{F}}_{p}\right) \cong \operatorname{Lie}\left(\mathcal{E} / \overline{\mathbb{F}}_{p}\right) \otimes_{\mathbb{Z}} \mathcal{O}_{F} \cong \overline{\mathbb{F}}_{p} \otimes_{\mathbb{Z}} \mathcal{O}_{F},
$$

so the characteristic polynomial condition on $\operatorname{Lie}\left(A / \overline{\mathbb{F}}_{p}\right)$ is satisfied. The triple $(A, \lambda, \rho)$ thus determines a point of $X$.

We have isomorphisms

$$
\operatorname{Hom}\left(\alpha_{p}, A[p]\right) \cong \operatorname{Hom}\left(\alpha_{p}, \mathcal{E}[p]\right) \otimes_{\mathbb{Z}} \mathcal{O}_{F} \cong \overline{\mathbb{F}}_{p} \otimes_{\mathbb{Z}} \mathcal{O}_{F},
$$

and the $p_{\tau}$ component of the latter is nonzero for all $\tau$. Thus $\operatorname{Hom}\left(\alpha_{p}, A[p]\right)_{p_{\tau}}$ is nonzero for all $\tau$, so $(A, \lambda, \rho)$ lies on $X_{\mathbb{S}}$. 


\section{Description of the sparse strata}

Now choose $\mathcal{V}$ so that $r_{\tau}(\mathcal{V})=s_{\tau}(\mathcal{V})=1$ for all $\tau$, and let $X=X_{U}(\mathcal{V})_{k_{0}}$. As we have seen, $X$ has a stratification whose closed strata $X_{S}$ are indexed by subsets $S$ of $\mathbb{S}$. We compute the global structure of $X_{S}$ for certain $S$ in terms of $U(2)$ Shimura varieties with different archimedean invariants.

Definition 4.1 A subset $S$ of $\mathbb{S}$ is sparse if for every $\tau$ in $S$, $\sigma \tau$ is not in $S$, where $\sigma$ is the Frobenius automorphism of $k_{0}$.

Remark 4.2 If $p$ splits completely in $F^{+}$, then there are no sparse sets $S$, and the theory of this section is vacuous. On the other hand, if the residue class degree of every prime of $F^{+}$above $p$ is even (so that in particular $d$ is even), then there exist sparse subsets of order up to $\frac{d}{2}$.

Fix a sparse subset $S$. Let $Y_{S}$ be the moduli space over $k_{0}$ whose points over $T$ are isomorphism classes of tuples $\left(A, \lambda, \rho, B, \lambda^{\prime}, \phi\right)$, where

1. $(A, \lambda, \rho)$ is a point of $X_{U}(T)$,

2. $B$ is an abelian scheme over $T$ of dimension $2 d$, with an action of $\mathcal{O}_{F}$,

3. $\lambda^{\prime}$ is a polarization of $B$, of degree prime to $p$, and

4. $\phi: A \rightarrow B$ is an $\mathcal{O}_{F}$-isogeny of degree $p^{2} d$, satisfying:

- $p \lambda=\phi^{\vee} \lambda^{\prime} \phi$

- for each $\tau$ not in $S$, the subbundle $\phi\left(H_{\mathrm{DR}}^{1}(B / T)\right)_{p_{\tau}}$ of $H_{\mathrm{DR}}^{1}(A / T)_{p_{\tau}}$ is equal to $H_{\mathrm{DR}}^{1}(A / T)_{p_{\tau}}$, and

- for each $\tau$ in $S$, the subbundle $\phi\left(H_{\mathrm{DR}}^{1}(B / T)\right)_{p_{\tau}}$ of $H_{\mathrm{DR}}^{1}(A / T)_{p_{\tau}}$ is simultaneously equal to both $\operatorname{Lie}(A / T)_{p_{\tau}}^{*}$ and $\operatorname{Fr}\left(H_{\mathrm{DR}}^{1}\left(A^{(p)} / T\right)_{p_{\tau}}\right)$, when considered as subbundles of $H_{\mathrm{DR}}^{1}(A / T)_{p_{\tau}}$. (Note that this implies that $(A, \lambda, \rho)$ is actually a point of $X_{S}$.)

The moduli space $Y_{S}$ can be thought of as parametrizing a point $(A, \lambda, \rho)$ of $X_{S}$, plus an isogeny $\phi: A \rightarrow B$ that serves as a "witness" to the fact that $(A, \lambda, \rho)$ lies in $X_{S}$. In fact, we will soon prove that the map $Y_{S} \rightarrow X_{S}$ that forgets $\phi$ is an isomorphism. First we need the following calculation:

Lemma 4.3 The rank of $\operatorname{Lie}(B / T)_{p_{\tau}}$ is two if $\tau$ is in $S$, zero if $\sigma \tau$ is in $S$, and one otherwise. In all cases, the rank of $\operatorname{Lie}(B / T)_{q_{\tau}}$ is equal to $2-$ $\operatorname{rank} \operatorname{Lie}(B / T)_{p_{\tau}}$.

Proof. Since $\operatorname{Lie}(B / T)_{p_{\tau}}$ is locally free, we can compute its rank after base change to a geometric point of $T$. We may thus assume $T=\operatorname{Spec} k$ for a perfect field $k$ containing $k_{0}$. This allows us to apply Dieudonné theory.

Let $\tilde{\mathcal{D}}_{A}$ (resp. $\tilde{\mathcal{D}}_{B}$ ) denote the Dieudonné module of the $p$-divisible group $A\left[p^{\infty}\right]$ (resp. $\left.B\left[p^{\infty}\right]\right)$. Let $\mathcal{D}_{A}$ (resp. $\mathcal{D}_{B}$ ) denote the Dieudonné module of $A[p]$ (resp. $B[p])$, so that $\mathcal{D}_{A} \cong \tilde{\mathcal{D}}_{A} / p \tilde{\mathcal{D}}_{A}$ and similarly for $\mathcal{D}_{B}$. 
The space $\operatorname{Lie}(B / T)_{p_{\tau}}^{*}$ is isomorphic to the image of $\left(\mathcal{D}_{B}\right)_{p_{\sigma \tau}}$ in $\left(\mathcal{D}_{B}\right)_{p_{\tau}}$ under Verschiebung. Thus we have:

$$
\begin{aligned}
\operatorname{dim}_{k} \operatorname{Lie}(B / T)_{p_{\tau}} & =2-\operatorname{dim}_{k}\left(\mathcal{D}_{B}\right)_{p_{\tau}} / V_{B}\left(\left(\mathcal{D}_{B}\right)_{p_{\sigma \tau}}\right) \\
& =2-\operatorname{dim}_{k}\left(\tilde{\mathcal{D}}_{B}\right)_{p_{\tau}} / V_{B}\left(\left(\tilde{\mathcal{D}}_{B}\right)_{p_{\sigma \tau}}\right)
\end{aligned}
$$

The map $\phi$ injects $\tilde{\mathcal{D}}_{B}$ into $\tilde{\mathcal{D}}_{A}$, so the latter is equal to

$$
2-\operatorname{dim}_{k} \phi\left(\tilde{\mathcal{D}}_{B}\right)_{p_{\tau}} / V_{A}\left(\phi\left(\tilde{\mathcal{D}}_{B}\right)_{p_{\sigma \tau}}\right) .
$$

We have the following equalities:

1. $\operatorname{dim}_{k}\left(\tilde{\mathcal{D}}_{A}\right)_{p_{\tau}} / \phi\left(\tilde{\mathcal{D}}_{B}\right)_{p_{\tau}}$ is one if $\tau$ is in $S$, and zero otherwise.

2. $\operatorname{dim}_{k}\left(\tilde{\mathcal{D}}_{A}\right)_{p_{\sigma \tau}} / \phi\left(\tilde{\mathcal{D}}_{B}\right)_{p_{\sigma \tau}}$ is one if $\sigma \tau$ is in $S$, and zero otherwise.

3. $\operatorname{dim}_{k}\left(\tilde{\mathcal{D}}_{A}\right)_{p_{\tau}} / V_{A}\left(\left(\tilde{\mathcal{D}}_{A}\right)_{p_{\sigma \tau}}\right)=1$.

The desired formula for the rank of $\operatorname{Lie}(B / T)_{p_{\tau}}$ follows immediately.

The Weil pairing induced by the polarization on $B$ identifies $\operatorname{Lie}(B / T)_{q_{\tau}}^{*}$ with $\left(\operatorname{Lie}(B / T)^{*}\right)_{p_{\tau}}^{\perp}$, where $\perp$ denotes orthogonal complement with respect to the Weil pairing

$$
H_{\mathrm{DR}}^{1}(B / T)_{p_{\tau}} \times H_{\mathrm{DR}}^{1}(B / T)_{q_{\tau}} \rightarrow \mathcal{O}_{T} .
$$

Thus we conclude that $\operatorname{Lie}(B / T)_{q_{\tau}}$ has rank equal to $2-\operatorname{rank} \operatorname{Lie}(B / T)_{p_{\tau}}$, for all $\tau$.

We are now in a position to show:

Proposition 4.4 The map $\pi: Y_{S} \rightarrow X_{S}$ is an isomorphism.

Proof. This map is clearly proper, and $X_{S}$ is smooth, so it suffices to show that $\pi$ is bijective on points and injective on tangent spaces.

Let $x$ be a closed point of $X_{S}$, with residue field $k_{x}$. Then $k_{x}$ is perfect, and $x$ corresponds to a tuple $(A, \lambda, \rho)$ in $X_{S}\left(k_{x}\right)$. We first construct a point of $Y_{S}$ lying over $x$.

Define a submodule $M$ of $H_{\mathrm{DR}}^{1}\left(A / k_{x}\right)$ by setting $M_{p_{\tau}}=H_{\mathrm{DR}}^{1}\left(A / k_{x}\right)_{p_{\tau}}$ for $\tau$ not in $S$, and $M_{p_{\tau}}=\operatorname{Lie}\left(A / k_{x}\right)_{p_{\tau}}^{*}$ for $\tau$ in $S$. Set $M_{q_{\tau}}=M_{p_{\tau}}^{\perp}$ for all $\tau$, where as usual $\perp$ denotes orthogonal complement with respect to the Weil pairing:

$$
H_{\mathrm{DR}}^{1}\left(A / k_{x}\right)_{p_{\tau}} \times H_{\mathrm{DR}}^{1}\left(A / k_{x}\right)_{q_{\tau}} \rightarrow k_{x} .
$$

Let $\mathcal{D}_{A}$ denote the Dieudonné module of $A[p]$. Then $\mathcal{D}_{A}$ is naturally isomorphic to $H_{\mathrm{DR}}^{1}\left(A / k_{x}\right)$. We consider $M$ as a subspace of $\mathcal{D}_{A}$ via this isomorphism. It is easy to verify that $M$ is stable under $F$ and $V$.

We have an exact sequence:

$$
0 \rightarrow M \rightarrow \mathcal{D}_{A} \rightarrow \mathcal{D}_{K} \rightarrow 0,
$$

where $\mathcal{D}_{K}$ is the Dieudonné module of some maximal isotropic subgroup scheme $K$ of $A[p]$. Let $B$ be the quotient $A / K$, and let $\phi$ be the natural map of $A$ onto $B$. Note that the submodule $\phi\left(H_{\mathrm{DR}}^{1}\left(B / k_{x}\right)\right)$ of $H_{\mathrm{DR}}^{1}\left(A / k_{x}\right)$ is simply $M$. 
Since $K$ is a maximal isotropic subgroup of $A[p]$, it follows that there is a prime-to- $p$ polarization $\lambda^{\prime}$ on $B$ such that $p \lambda=\phi^{\vee} \lambda^{\prime} \phi$. Thus $\left(A, \lambda, \rho, B, \lambda^{\prime}, \phi\right)$ is a point of $Y_{S}$ mapping to $x$.

On the other hand, if $\left(A, \lambda, \rho, B, \lambda_{1}, \phi\right)$ is any point of $Y_{S}$ mapping to $x$, then by definition $\phi\left(H_{\mathrm{DR}}^{1}\left(B / k_{x}\right)\right)$ is equal to $M$. Thus the kernel of $\phi$ is equal to $K$, and hence this tuple is isomorphic to the tuple constructed above. In particular, $\pi$ is a bijection on closed points.

It now suffices to show that the map $\pi$ is an injection on tangent spaces at every closed point.

Let $x$ be a closed point of $Y_{S}$, with (necessarily perfect) residue field $k_{x}$. The point $x$ corresponds to a tuple $\left(A, \lambda, \rho, B, \lambda^{\prime}, \phi\right)$. Let $\mathbb{I}$ denote the scheme Spec $k_{x}[\epsilon] / \epsilon^{2}$. As in the proof of Proposition 3.4, specifying a tangent vector to $X$ at $\pi(x)$ is equivalent to specifying, for each $\tau$, a lift of $\operatorname{Lie}\left(A / k_{x}\right)_{p_{\tau}}^{*}$ to a subbundle $\omega_{A, \tau}$ of $\left(H_{\text {cris }}^{1}\left(A / k_{x}\right)_{\mathbb{I}}\right)_{p_{\tau}}$.

Fix choices of $\omega_{A, \tau}$ corresponding to a fixed tangent vector $v$ to $X_{S}$ at $x$, and let $\tilde{A}$ be the abelian scheme over $\mathbb{I}$ corresponding to this tangent vector. To lift this tangent vector to a tangent vector to $Y_{S}$ at $x$, we must specify a lift $\tilde{B}$ of $B$, a lift of the polarization $\lambda^{\prime}$ on $B$, and a lift of the isogeny $A \rightarrow B$.

By Theorem 3.5, to specify $\tilde{B}$, we must specify for each $\tau$ lifts $\omega_{B, \tau}$ of $\operatorname{Lie}\left(B / k_{x}\right)_{p_{\tau}}^{*}$ and $\omega_{B, \tau}^{\prime}$ of $\operatorname{Lie}\left(B / k_{x}\right)_{q_{\tau}}^{*}$ to $\left(H_{\text {cris }}^{1}\left(B / k_{x}\right)_{\mathbb{I}}\right)_{p_{\tau}}$ and $\left(H_{\text {cris }}^{1}\left(B / k_{x}\right)_{\mathbb{I}}\right)_{q_{\tau}}$, respectively. The polarization $\lambda^{\prime}$ will lift to $\tilde{B}$ if and only if $\omega_{B, \tau}^{\prime}=\omega_{B, \tau}^{\perp}$ for all $\tau$, where $\perp$ denotes orthogonal complement under the pairing

$$
\left(H_{\text {cris }}^{1}\left(B / k_{x}\right)_{\mathbb{I}}\right)_{p_{\tau}} \times\left(H_{\text {cris }}^{1}\left(B / k_{x}\right)_{\mathbb{I}}\right)_{q_{\tau}} \rightarrow k_{x}[\epsilon] / \epsilon^{2} .
$$

Moreover, if a lift exists at all, it will be unique.

The map $\phi: A \rightarrow B$ will lift to a map $\tilde{A} \rightarrow \tilde{B}$ if and only if $\phi\left(\omega_{B, \tau}\right) \subset \omega_{A, \tau}$ and $\phi\left(\omega_{B, \tau}^{\prime}\right) \subset \omega_{A, \tau}^{\perp}$ for all $\tau$. Again, if such a lift exists it will be unique.

Thus specifying a lift of $v$ to a tangent vector to $Y_{S}$ at $x$ is equivalent to specifying, for each $\tau$, an $\omega_{B, \tau}$ such that $\phi\left(\omega_{B, \tau}\right) \subset \omega_{A, \tau}$.

Suppose first that $\tau$ is in $S$. Then Lemma 4.3 implies that the rank of $\operatorname{Lie}\left(B / k_{x}\right)_{p_{\tau}}^{*}$ is two. In particular, it is all of $H_{\mathrm{DR}}^{1}\left(B / k_{x}\right)_{p_{\tau}}$. Thus the only possible choice for $\omega_{B, \tau}$ is all of $\left(H_{\text {cris }}^{1}\left(B / k_{x}\right)_{\mathbb{I}}\right)_{p_{\tau}}$.

Suppose that $\sigma \tau$ is in $S$. Then $\tau$ is not in $S$, as $S$ is sparse, and Lemma 4.3 implies that $\operatorname{Lie}\left(B / k_{x}\right)_{p_{\tau}}^{*}=0$. Thus the only possible choice for $\omega_{B, \tau}$ is the zero module.

Finally, suppose that neither $\tau$ nor $\sigma \tau$ is in $S$. Then $\operatorname{Lie}\left(B / k_{x}\right)_{p_{\tau}}^{*}$ has rank one by Lemma 4.3. so $\omega_{B, \tau}$ must have rank one, and map to $\omega_{A, \tau}$ under $f$. But as $\tau$ is not in $S, \phi$ induces an isomorphism

$$
\left(H_{\text {cris }}^{1}\left(B / k_{x}\right)_{\mathbb{I}}\right)_{p_{\tau}} \rightarrow\left(H_{\text {cris }}^{1}\left(A / k_{x}\right)_{\mathbb{I}}\right)_{p_{\tau}} .
$$

Moreover, $\omega_{A, \tau}$ has rank one. Thus $\omega_{A, \tau}$ determines $\omega_{B, \tau}$ uniquely.

It follows that the tangent space to $Y_{S}$ at $x$ injects into the tangent space to $X_{S}$ at $\pi(x)$. Hence $\pi$ is proper, bijective on points, and injective on tangent spaces, and is therefore an isomorphism. 
The isomorphism $Y_{S} \rightarrow X_{S}$ allows us to associate to every triple $(A, \lambda, \rho) \in$ $X_{S}$ a canonical isogeny $f: A \rightarrow B$, where $B$ is an abelian scheme over $T$ with $\mathcal{O}_{F}$ action and polarization $\lambda^{\prime}$. The pair $\left(B, \lambda^{\prime}\right)$ is "almost" a point of a $U(2)$ Shimura variety; the only thing missing is a level structure $\rho^{\prime}$. We cannot define such a level structure in terms of $\mathcal{V}$, because $r_{\tau}(\mathcal{V})=1$ for all $\tau$, but, for instance, $\operatorname{Lie}(B / T)_{p_{\tau}}$ has dimension 2 if $\tau$ is in $S$.

Define $r_{\tau}^{\prime}$ and $s_{\tau}^{\prime}$ via:

1. $r_{\tau}^{\prime}=2$ if $\tau \in S$.

2. $r_{\tau}^{\prime}=0$ if $\sigma \tau \in S$.

3. $r_{\tau}^{\prime}=1$ if neither $\tau$ nor $\sigma \tau$ lies in $S$.

4. $s_{\tau}^{\prime}=2-r_{\tau}^{\prime}$ for all $\tau$.

Then, by Lemma 4.3, $\operatorname{Lie}(B / T)_{p_{\tau}}$ has rank $r_{\tau}^{\prime}$ for all $\tau$, and $\operatorname{Lie}(B / T)_{q_{\tau}}$ has rank $s_{\tau}^{\prime}$ for all $\tau$. Thus if we wish to make $B$ into a point on some $U(2)$ Shimura variety $X_{U}\left(\mathcal{V}^{\prime}\right)$, we must have $r_{\tau}\left(\mathcal{V}^{\prime}\right)=r_{\tau}^{\prime}$ for all $\tau$.

Fix an $F$-vector space $\mathcal{V}^{\prime}$ with a pairing $\langle,\rangle^{\prime}$ satisfying the conditions of section 2 such that $r_{\tau}\left(\mathcal{V}^{\prime}\right)=r_{\tau}^{\prime}$ for all $\tau$, and such that there is an isomorphism $\mathcal{V}^{\prime} \otimes \mathbb{A}_{\mathbb{Q}}^{f} \cong \mathcal{V} \otimes \mathbb{A}_{\mathbb{Q}}^{f}$ taking $\langle,\rangle^{\prime}$ to $\langle$,$\rangle . (The existence of such a vector space is$ a consequence of Corollary 8.2 , which is proven in the appendix, or of Proposition 8.1 if we are working more generally with $X_{U}^{D}(\mathcal{V})$ rather than $X_{U}(\mathcal{V})$.) Fix in addition a particular isomorphism $\psi$ of $\mathcal{V}^{\prime} \otimes \mathbb{A}_{\mathbb{Q}}^{f}$ with $\mathcal{V} \otimes \mathbb{A}_{\mathbb{Q}}^{f}$, and let $\mathcal{T}^{\prime}=\psi(\mathcal{T})$. Let $G^{\prime}$ denote the unitary group associated to $\mathcal{V}^{\prime}$ and $\langle,\rangle^{\prime}$. The map $\psi$ then defines an isomorphism $G\left(\mathbb{A}_{\mathbb{Q}}^{f}\right) \rightarrow G^{\prime}\left(\mathbb{A}_{\mathbb{Q}}^{f}\right)$, and we denote by $U^{\prime}$ the subgroup of $G^{\prime}\left(\mathbb{A}_{\mathbb{Q}}^{f}\right)$ that is the image of $U$ under this isomorphism. Then $\psi$, the isogeny $f: A \rightarrow B$, and the $U$-level structure $\rho$ on $A$ induce a unique $U^{\prime}$-level structure $\rho^{\prime}$ on $B$. In particular, the triple $\left(B, \lambda^{\prime}, \rho^{\prime}\right)$ is a point of $X^{\prime}=X_{U^{\prime}}\left(\mathcal{V}^{\prime}\right)_{k_{0}}$. We thus obtain a map $X_{S} \rightarrow X^{\prime}$.

The scheme $X_{S}$ has dimension $d-\# S$, whereas we have:

$$
\operatorname{dim} X^{\prime}=\sum_{\tau} r_{\tau}^{\prime} s_{\tau}^{\prime}=d-2 \# S
$$

Thus this map has nontrivial fibers, of dimension at least equal to the order of $S$.

In order to recover $(A, \lambda, \rho)$ given $\left(B, \lambda^{\prime}, \rho^{\prime}\right)$, we need to remember some extra data. Let $\left(A, \lambda, \rho, B, \lambda^{\prime}, \phi\right)$ be the point of $Y_{S}$ associated to $(A, \lambda, \rho)$. Then the kernel of $\phi$ is contained in $A[p]$, so multiplication by $p$ factors through $\phi$. We thus obtain an isogeny $\phi^{\prime}: B \rightarrow A$, of degree $p^{2 d}$.

We make the following observations, which are clear::

- The kernel of $\phi^{\prime}$ is contained in $B[p]$.

- We have $p \lambda^{\prime}=\left(\phi^{\prime}\right)^{\vee} \lambda \phi^{\prime}$.

- $\phi^{\prime}\left(H_{\mathrm{DR}}^{1}(A / T)_{p_{\tau}}\right)$ has rank one for $\tau$ in $S$, and is zero for $\tau$ outside $S$. 
Let $Y_{S}^{\prime}$ denote the moduli space that sends a $k_{0}$-scheme $T$ to the set of isomorphism classes of tuples $\left(B, \lambda^{\prime}, \rho^{\prime}, A, \lambda, \phi^{\prime}\right)$, where

1. $\left(B, \lambda^{\prime}, \rho^{\prime}\right)$ is a point of $X^{\prime}(T)$,

2. $A$ is an abelian scheme over $T$ of dimension $2 d$, with an action of $\mathcal{O}_{F}$,

3. $\lambda$ is a polarization of $A$, of degree prime to $p$, and

4. $\phi^{\prime}: B \rightarrow A$ is an $\mathcal{O}_{F}$-isogeny, such that:

- $p \lambda^{\prime}=\left(\phi^{\prime}\right)^{\vee} \lambda \phi^{\prime}$, and

- for each $\tau, \phi^{\prime}\left(H_{\mathrm{DR}}^{1}(A / T)_{p_{\tau}}\right)$ is locally free of rank one if $\tau$ is in $S$, and zero otherwise.

The construction outlined above defines a map $Y_{S} \rightarrow Y_{S}^{\prime}$. Let us now define an inverse map. Suppose we have a point $\left(B, \lambda^{\prime}, \rho^{\prime}, A, \lambda, \phi^{\prime}\right)$ of $Y_{S}^{\prime}$. As before, the $U^{\prime}$-level structure on $B$ induces a unique $U$-level structure $\rho$ on $A$, and hence a unique point $(A, \lambda, \rho)$ of $X$. Factoring multiplication by $p$ on $B$ through $\phi^{\prime}$ gives us an isogeny $\phi: A \rightarrow B$, of degree $p^{2 d}$.

Lemma 4.5 The tuple $\left(A, \lambda, \rho, B, \lambda^{\prime}, \phi\right)$ defines a point of $Y_{S}$ that maps to $\left(B, \lambda^{\prime}, p \rho^{\prime}, A, \lambda, \phi^{\prime}\right)$ under the map $Y_{S} \rightarrow Y_{S}^{\prime}$. Thus $Y_{S}$ is isomorphic to $Y_{S}^{\prime}$.

Proof. Exactly as above, we see that the kernel of $\phi$ is contained in $A[p]$, that $p \lambda=\phi^{\vee} \lambda^{\prime} \phi$, and that $\phi\left(H_{\mathrm{DR}}^{1}(B / T)_{p_{\tau}}\right)$ has rank one if $\tau$ is in $S$ and rank two otherwise. It remains to show that for $\tau \in S$, we have

$$
\phi\left(H_{\mathrm{DR}}^{1}(B / T)_{p_{\tau}}\right)=\operatorname{Lie}(A / T)_{p_{\tau}}^{*},
$$

and also

$$
\phi\left(H_{\mathrm{DR}}^{1}(B / T)_{p_{\tau}}\right)=\operatorname{Fr}\left(H_{\mathrm{DR}}^{1}\left(A^{(p)} / T\right)_{p_{\tau}}\right) .
$$

Note that for $\tau$ in $S, \operatorname{Lie}(A / T)_{p_{\tau}}^{*}$ has rank one, $\operatorname{Lie}(B / T)_{p_{\tau}}^{*}$ has rank two, and the kernel of the map

$$
H_{\mathrm{DR}}^{1}(B / T)_{p_{\tau}} \rightarrow H_{\mathrm{DR}}^{1}(A / T)_{p_{\tau}}
$$

has rank one. Thus $\phi$ induces a surjection of $\operatorname{Lie}(B / T)_{p_{\tau}}^{*}$ (equivalently, of $\left.H_{\mathrm{DR}}^{1}(B / T)_{p_{\tau}}\right)$ onto $\operatorname{Lie}(A / T)_{p_{\tau}}^{*}$.

On the other hand, as $\phi$ commutes with relative Frobenius, we have

$$
\phi\left(\operatorname{Fr}\left(H_{\mathrm{DR}}^{1}\left(B^{(p)} / T\right)_{p_{\tau}}\right)\right) \subset \operatorname{Fr}\left(H_{\mathrm{DR}}^{1}\left(A^{(p)} / T\right)_{p_{\tau}}\right) .
$$

For $\tau \in S, \operatorname{Fr}\left(H_{\mathrm{DR}}^{1}\left(B^{(p)} / T\right)_{p_{\tau}}\right)$ has rank two, $\operatorname{Fr}\left(H_{\mathrm{DR}}^{1}\left(A^{(p)} / T\right)_{p_{\tau}}\right)$ has rank one, and the kernel of $\phi$ has rank one, so again $\phi$ induces a surjection of $H_{\mathrm{DR}}^{1}(B / T)_{p_{\tau}}$ onto $\operatorname{Fr}\left(H_{\mathrm{DR}}^{1}\left(A^{(p)} / T\right)_{p_{\tau}}\right)$.

This construction defines a map $Y_{S}^{\prime} \rightarrow Y_{S}$ that is nearly an inverse to the map $Y_{S} \rightarrow Y_{S}^{\prime}$ defined earlier. (The composition of the two maps is the automorphism of $Y_{S}$ which multiplies the level structure $\rho$ by $p$.) We thus obtain an isomorphism $Y_{S} \rightarrow Y_{S}^{\prime}$.

Let $Z_{S}$ be the moduli space of tuples $\left(B, \lambda^{\prime}, \rho^{\prime}, M\right)$, where $\left(B, \lambda^{\prime}, \rho^{\prime}\right)$ is a point of $X^{\prime}(T)$, and $M$ is an $\mathcal{O}_{F}$-stable subbundle of $H_{\mathrm{DR}}^{1}(B / T)$ such that: 
1. $M_{p_{\tau}}$ is locally free of rank one for $\tau$ in $S$, and zero for $\tau$ not in $S$, and

2. $M_{q_{\tau}}=M_{p_{\tau}}^{\perp}$, where $\perp$ is with respect to the Weil pairing

$$
H_{\mathrm{DR}}^{1}(B / T)_{p_{\tau}} \times H_{\mathrm{DR}}^{1}(B / T)_{q_{\tau}} \rightarrow \mathcal{O}_{T} .
$$

Let $\mathcal{B}$ denote the universal abelian scheme on $X^{\prime}$. Then $Z_{S}$ is simply the fiber product, over $X^{\prime}$, of the bundles $P\left(H_{\mathrm{DR}}^{1}\left(\mathcal{B} / X^{\prime}\right)_{p_{\tau}}\right)$, for $\tau \in S$.

We have a natural map $Y_{S}^{\prime} \rightarrow Z_{S}$ that takes the point $\left(B, \lambda^{\prime}, \rho^{\prime}, A, \lambda, \phi^{\prime}\right)$ of $Y_{S}^{\prime}$ to the point $\left(B, \lambda^{\prime}, \rho^{\prime}, \phi^{\prime}\left(H_{\mathrm{DR}}^{1}(A / T)\right)\right)$ of $Z_{S}$.

Proposition 4.6 The natural map $Y_{S}^{\prime} \rightarrow Z_{S}$ is a bijection on points.

Proof. We construct an inverse map on the level of points. Let $k$ be a perfect field containing $k_{0}$, and let $\left(B, \lambda^{\prime}, \rho^{\prime}, M\right)$ be a $k$-valued point of $Z_{S}$.

Let $\mathcal{D}_{B}$ denote the Dieudonné module of $B[p]$, and consider $M$ as a subspace of $\mathcal{D}_{B}$. Then $M$ is stable under $F$ and $V$, as for $\tau$ in $S, F\left(\left(\mathcal{D}_{B}\right)_{p_{\tau}}\right)$ and $V\left(\left(\mathcal{D}_{B}\right)_{p_{\tau}}\right)$ both vanish. Hence we have an exact sequence of Dieudonné modules:

$$
0 \rightarrow M \rightarrow \mathcal{D}_{B} \rightarrow \mathcal{D}_{K} \rightarrow 0,
$$

where $\mathcal{D}_{K}$ is the Dieudonné module of some finite flat group scheme $K$. We thus obtain an injection of $K$ into $B[p]$.

Let $A=B / K$, and let $\phi^{\prime}: B \rightarrow A$ be the quotient map. Since $K$ is a maximal isotropic subgroup of $B[p]$, there is a prime-to- $p$ polarization $\lambda$ on $A$ such that $p \lambda^{\prime}=\left(\phi^{\prime}\right)^{\vee} \lambda \phi^{\prime}$. Then $\left(B, \lambda^{\prime}, \rho^{\prime}, A, \lambda, \phi^{\prime}\right)$ is a point of $Y_{S}^{\prime}$, and it is easy to see that this gives an inverse to the map $Y_{S}^{\prime} \rightarrow Z_{S}$ defined above.

Composing the map $Y_{S}^{\prime} \rightarrow Z_{S}$ with the sequence of isomorphisms $X_{S} \cong$ $Y_{S} \cong Y_{S}^{\prime}$ yields a map $X_{S} \rightarrow Z_{S}$ that is a bijection on points. (In general this map fails to be a geometric isomorphism.)

Definition 4.7 A map $Y \rightarrow Z$ of $k$-schemes is a Frobenius factor if there exists a map $f^{\prime}: Z_{p^{r}} \rightarrow Y$ such that $f f^{\prime}: Z_{p^{r}} \rightarrow Z$ is the rth power of the Frobenius morphism. (Here $Z_{p^{r}}$ is the $k$-scheme obtained from $Z$ by composing the structure map $Z \rightarrow$ Spec $k$ with the $p^{r}$-th power Frobenius automorphism of Spec $k$.)

Note that Frobenius factors are isomorphisms on étale cohomology.

Proposition 4.8 Let $Y$ and $Z$ be schemes of finite type over a perfect field $k$ of characteristic $p$, such that $Z$ is normal and $Y$ is reduced. Let $f: Y \rightarrow Z$ be a proper morphism that is a bijection on points. Then $f$ is a Frobenius factor.

Proof. As $f$ is a bijection on points, $K(Y)$ is a purely inseparable extension of $K(Z)$. Thus for $r$ sufficiently large, every $p^{r}$-th power of an element of $K(Y)$ lies in $K(Z)$. In particular, we have $K(Y) \subset K\left(Z_{p^{r}}\right)$, For each $U \subset Z$ in an affine cover, $\mathcal{O}_{Z_{p^{r}}}(U)$ is the integral closure of $\mathcal{O}_{Z}(U)$ in $K\left(Z_{p^{r}}\right)$. Since $f: Y \rightarrow Z$ is finite, $\mathcal{O}_{Y}(U)$ is integral over $\mathcal{O}_{Z}(U)$, and hence contained in $\mathcal{O}_{Z_{p^{r}}}(U)$. These inclusions give the map $f^{\prime}: Z_{p^{r}} \rightarrow Y$, as required.

Combining this with the preceding result, we obtain: 
Theorem 4.9 The natural map $X_{S} \rightarrow Z_{S}$ is a Frobenius factor.

We will have need of the following lemma in the next section:

Lemma 4.10 Let $\mathcal{A}_{S}$ be the universal abelian scheme on $X_{S}$. Then for all $\tau \notin S$, the bundle $H_{\mathrm{DR}}^{1}\left(\mathcal{A}_{S} / X_{S}\right)_{p_{\tau}}$ is the pullback of $H_{\mathrm{DR}}^{1}\left(\mathcal{B} / X^{\prime}\right)_{p_{\tau}}$ under the natural map $X_{S} \rightarrow X^{\prime}$ defined above.

Proof. Let $\left(\mathcal{A}_{S}, \lambda, \rho, \mathcal{B}_{S}, \lambda^{\prime}, \phi_{S}\right)$ be the universal object of $Y_{S}$. Then $\phi_{S}$ induces an isomorphism:

$$
\phi_{S}: H_{\mathrm{DR}}^{1}\left(\mathcal{A}_{S} / X_{S}\right)_{p_{\tau}} \cong H_{\mathrm{DR}}^{1}\left(\mathcal{B}_{S} / X_{S}\right)_{p_{\tau}}
$$

for all $\tau$ outside $S$.

On the other hand, by the definition of the natural map $X_{S} \rightarrow X^{\prime}$, we have $\mathcal{B}_{S} \cong \mathcal{B} \times_{X^{\prime}} X_{S}$. Thus $H_{\mathrm{DR}}^{1}\left(\mathcal{B}_{S} / X_{S}\right)_{p_{\tau}}$ is naturally isomorphic to the pullback of $H_{\mathrm{DR}}^{1}\left(\mathcal{B} / X^{\prime}\right)_{p_{\tau}}$ for all $\tau$.

\section{$5 \quad \Gamma_{0}(p)$ level structures}

We now turn to the reduction of $U(2)$ Shimura varieties with level structure at $p$. In particular fix a $\mathcal{V}$ such that $r_{\tau}(\mathcal{V})=s_{\tau(\mathcal{V})}=1$ for all $\tau$, and consider the Shimura variety $X_{U, p}(\mathcal{V})$ defined in section 2 Let $\mathcal{A}$ and $\mathcal{B}$ be the universal abelian schemes on $X_{U, p}(\mathcal{V})$, and let $\phi_{\text {univ }}: \mathcal{A} \rightarrow \mathcal{B}$ denote the universal isogeny between them. Multiplication by $p$ on $\mathcal{A}$ factors through $\phi_{\text {univ }}$, and we denote by $\phi_{\text {univ }}^{\prime}$ the isogeny from $\mathcal{B}$ to $\mathcal{A}$ obtained in this manner.

Let $\mathcal{E}_{p_{\tau}}$ and $\mathcal{E}_{p_{\tau}}^{\prime}$ be the bundles $\operatorname{Lie}\left(\mathcal{A} / X_{U, p}(\mathcal{V})\right)_{p_{\tau}}^{*}$ and $\operatorname{Lie}\left(\mathcal{B} / X_{U, p}(\mathcal{V})\right)_{p_{\tau}}^{*}$, respectively. For all $\tau, \phi_{\text {univ }}$ and $\phi_{\text {univ }}^{\prime}$ can be thought of as sections of $\operatorname{Hom}\left(\mathcal{E}_{p_{\tau}}^{\prime}, \mathcal{E}_{p_{\tau}}\right)$ and $\operatorname{Hom}\left(\mathcal{E}_{p_{\tau}}, \mathcal{E}_{p_{\tau}}^{\prime}\right)$, which we denote by $h_{\tau}$ and $h_{\tau}^{\prime}$, respectively.

Note that the composition of $h_{\tau}$ and $h_{\tau}^{\prime}$ in either direction is multiplication by $p$, because the same holds for $\phi_{\text {univ }}$ and $\phi_{\text {univ }}^{\prime}$. Thus in particular on any point $x$ of the fiber of $X_{U, p}(\mathcal{V})$ over $p$, either $h_{\tau}$ or $h_{\tau}^{\prime}$ vanishes.

For subsets $S$ and $S^{\prime}$ of $\mathbb{S}$, define the subscheme $X_{U, p}(\mathcal{V})_{S, S^{\prime}}$ of $X_{U, p}(\mathcal{V})_{k_{0}}$ to be the common zeros of the $h_{\tau}$ for $\tau \in S$ and the $h_{\tau}^{\prime}$ for $\tau \in S^{\prime}$. Then $X_{U, p}(\mathcal{V})_{k_{0}}$ is the union of the $X_{U, p}(\mathcal{V})_{S, S^{\prime}}$ for pairs of sets $S, S^{\prime}$ with $S \cup S^{\prime}=\mathbb{S}$.

We study these subschemes by constructing a local model for $X_{U, p}(\mathcal{V})$ in which the above subschemes have natural analogues. Constructing such models is standard; the technique is due originally to de Jong $\mathrm{dJ}$. The result we need is a special case of results of Rapoport-Zink [RZ1, Appendix.

For each $\tau$, let $M_{\tau}$ and $M_{\tau}^{\prime}$ be free $W\left(\mathbb{F}_{p}\right)$ modules of rank two, with bases $\left\{e_{1, \tau}, e_{2, \tau}\right\}$ and $\left\{e_{1, \tau}^{\prime}, e_{2, \tau}^{\prime}\right\}$, respectively. Let $\alpha_{\tau}: M_{\tau} \rightarrow M_{\tau}^{\prime}$ be defined by $\alpha_{\tau}\left(e_{1, \tau}\right)=e_{1, \tau}^{\prime}$ and $\alpha_{\tau}\left(e_{2, \tau}\right)=p e_{2, \tau}^{\prime}$. Similarly, define $\alpha_{\tau}^{\prime}$ by $\alpha_{\tau}^{\prime}\left(e_{1, \tau}^{\prime}\right)=p e_{1, \tau}$ and $\alpha_{\tau}^{\prime}\left(e_{2, \tau}^{\prime}\right)=e_{2, \tau}$.

Define $\tilde{X}_{U, p}(\mathcal{V})$ to be the scheme representing the functor parameterizing tuples $\left(A, \lambda, \rho, B, \lambda^{\prime}, \phi,\left\{e_{\tau}\right\},\left\{e_{\tau}^{\prime}\right\}\right)$, where

1. $\left(A, \lambda, \rho, B, \lambda^{\prime}, \phi\right)$ is a point of $X_{U, p}(\mathcal{V})(T)$, 
2. for each $\tau, e_{\tau}$ and $e_{\tau}^{\prime}$ are isomorphisms of $H_{\mathrm{DR}}^{1}(B / T)_{p_{\tau}}$ with $M_{\tau} \otimes_{W\left(k_{0}\right)} T$ and $H_{\mathrm{DR}}^{1}(A / T)_{p_{\tau}}$ with $M_{\tau}^{\prime} \otimes_{W\left(k_{0}\right)} T$, respectively.

3. We require that under the isomorphism $e_{\tau}$ and $e_{\tau}^{\prime}$, the map

$$
\phi: H_{\mathrm{DR}}^{1}(B / T)_{p_{\tau}} \rightarrow H_{\mathrm{DR}}^{1}(A / T)_{p_{\tau}}
$$

corresponds to $\alpha$, and the map

$$
\phi^{\prime}: H_{\mathrm{DR}}^{1}(A / T)_{p_{\tau}} \rightarrow H_{\mathrm{DR}}^{1}(B / T)_{p_{\tau}}
$$

corresponds to $\alpha^{\prime}$. (Here $\phi^{\prime}: B \rightarrow A$ is the map obtained by factoring multiplication by $p$ through $\phi$.)

It is then standard that the natural map to $\tilde{X}_{U, p}(\mathcal{V}) \rightarrow X_{U, p}(\mathcal{V})$ is smooth and surjective.

We now describe the local model for $X_{U, p}$. Let $\mathcal{M}$ denote the $W\left(k_{0}\right)$-scheme parameterizing tuples $\left(\left\{V_{\tau}, V_{\tau}^{\prime}\right\}\right)$, where for each $\tau, V_{\tau}$ is a rank one subbundle of $\left(M_{\tau}\right)_{T}, V_{\tau}^{\prime}$ is a rank one subbundle of $\left(M_{\tau}^{\prime}\right)_{T}, \alpha_{\tau}$ maps $V$ to $V^{\prime}$, and $\alpha_{\tau}^{\prime}$ maps $V^{\prime}$ to $V$. The scheme $\mathcal{M}$ is the product of schemes $\mathcal{M}_{\tau}$ paramaterizing $\left(V_{\tau}, V_{\tau}^{\prime}\right)$ as above for a single $\tau$.

The general fiber of $\mathcal{M}_{\tau}$ is isomorphic to $\mathbb{P}^{1}$, while the fiber of $\mathcal{M}_{\tau}$ over $\mathbb{F}_{p}$ is the union of two components. More precisely, let $\beta_{\tau}, \beta_{\tau}^{\prime}$ be the maps $V_{\tau} \rightarrow V_{\tau}^{\prime}$ and $V_{\tau}^{\prime} \rightarrow V_{\tau}$ induced by $\alpha_{\tau}$ and $\alpha_{\tau}^{\prime}$, respectively. These are sections of tautological bundles on $\mathcal{M}_{\tau}$. Their composition is multiplication by $p$, and the special fiber of $\mathcal{M}_{\tau}$ is thus the union of $Z\left(\alpha_{\tau}\right)$ and $Z\left(\beta_{\tau}\right)$. Each of these components is isomorphic to $\mathbb{P}_{\mathbb{F}_{p}}^{1}$.

For $S$ and $S^{\prime}$ such that $S \cup S^{\prime}=\mathbb{S}$, define $\mathcal{M}_{S, S^{\prime}}$ to be the zero locus of $\beta_{\tau}$ for $\tau \in S$ and $\beta_{\tau}^{\prime}$ for $\tau \in S^{\prime}$.

We have a map $g: \tilde{X}_{U, p}(\mathcal{V}) \rightarrow \mathcal{M}$ that takes $\left(A, \lambda, \rho, B, \lambda^{\prime}, \phi,\left\{e_{\tau}\right\},\left\{e_{\tau}^{\prime}\right\}\right)$ to $\left(\left\{e_{\tau}\left(\operatorname{Lie}(B / T)_{p_{\tau}}^{*}\right), e_{\tau}^{\prime}\left(\operatorname{Lie}(A / T)_{p_{\tau}}^{*}\right)\right\}\right)$. The arguments of [RZ1] show that $g$ is smooth, and hence (via dJ], corollary 4.6) that every point $x$ of $X_{U, p}(\mathcal{V})$ has an étale neighborhood that is isomorphic to an étale neighborhood of some point $w$ of $\mathcal{M}$. Moreover, the pullbacks to $\tilde{X}_{U, p}(\mathcal{V})$ of $\beta_{\tau}$ and $\beta_{\tau}^{\prime}$ coincide with the pullbacks of $h_{\tau}$ and $h_{\tau}^{\prime}$. Thus if $x$ lies in $X_{U, p}(\mathcal{V})_{S, S^{\prime}}$, then $w$ can be taken to be inside $\mathcal{M}_{S, S^{\prime}}$. The following corollaries are immediate.

Corollary $5.1 X_{U, p}(\mathcal{V})_{k_{0}}$ is reduced.

Corollary 5.2 Let $S, S^{\prime} \subset \mathbb{S}$ satisfy $S \cup S^{\prime}=\mathbb{S}$. Then $X_{U, p}(\mathcal{V})_{S, S^{\prime}}$ is a smooth subscheme of $X_{U, p}(\mathcal{V})_{k_{0}}$, of codimension equal to $\#\left(S \cap S^{\prime}\right)$. In particular, if $S$ and $S^{\prime}$ are disjoint then $X_{U, p}(\mathcal{V})_{S, S^{\prime}}$ is a disjoint union of irreducible components of $X_{U, p}(\mathcal{V})$. Moreover, every irreducible component of $\left(X_{U, p}\right)_{k_{0}}$ is smooth.

Corollary 5.3 Let $x$ be a closed point of the fiber of $X_{U, p}(\mathcal{V})$. Let $S$ be the set of all $\tau$ such that $h_{\tau}(x)=0$, and let $S^{\prime}$ be the set of all $\tau$ such that $h_{\tau}^{\prime}(x)=0$. Then the completion of the strict henselization of the local ring of $X_{U, p}(\mathcal{V})$ at $x$ is isomorphic to:

$$
W\left(\overline{\mathbb{F}}_{p}\right)\left[\left[X_{\tau}: \tau \in S, Y_{\tau}: \tau \in S^{\prime}\right]\right] /\left(X_{\tau} Y_{\tau}-p: \tau \in S \cap S^{\prime}\right) .
$$


Remark 5.4 In visualizing the way in which the subvarieties $X_{U, p}(\mathcal{V})_{S, S^{\prime}}$ fit together, it is helpful to consider them as corresponding to various faces of the cube $[0,1]^{\mathbb{S}}$. Let $X_{U, p}(\mathcal{V})_{S, S^{\prime}}$ correspond to the face consisting of points whose $\tau$ coordinate is zero for all $\tau$ in $S$ but not in $S^{\prime}$, and one for all $\tau$ in $S^{\prime}$ but not in $S$. Then the dimension of any face of this cube is equal to the codimension of the corresponding subvariety. Moreover, if $X_{1}, \ldots, X_{r}$ is a collection of subschemes of the above form, corresponding to faces $F_{1}, \ldots, F_{r}$ of the cube, then the intersection of the $X_{i}$ is also of the above form, and corresponds to the smallest face of $[0,1]^{\mathbb{S}}$ containing all of the $F_{i}$.

Remark 5.5 Strictly speaking, we have only proven that $X_{U, p}(\mathcal{V})_{S, S^{\prime}}$ has the above properties if it is nonempty. This nonemptiness is a consequence of our characterization of the closed points on $X_{U, p}(\mathcal{V})_{S, S^{\prime}}$, below.

Now that we have an understanding of the local structure of the special fiber, we turn to the global structure of the subvarieties $X_{U, p}(\mathcal{V})_{S, S^{\prime}}$. The key is the following observation:

Proposition 5.6 Let $k$ be a perfect field of characteristic $p$, and $x$ a $k$-valued point of $X_{U, p}(\mathcal{V})$, corresponding to an isogeny $\phi_{x}: A / k \rightarrow B / k$. Then:

1. $h_{\tau}^{\prime}(x)=0$ if and only if $\phi_{x}\left(H_{\mathrm{DR}}^{1}(B / k)\right)_{p_{\tau}}=\operatorname{Lie}(A / k)_{p_{\tau}}^{*}$, and

2. $h_{\tau}(x)=0$ if and only if $\phi_{x}\left(H_{\mathrm{DR}}^{1}(B / k)\right)_{p_{\sigma \tau}}=\operatorname{Fr}\left(H_{\mathrm{DR}}^{1}\left(A^{(p)} / k\right)\right)_{p_{\sigma \tau}}$, where Fr is the relative Frobenius $A \rightarrow A^{(p)}$.

Proof. Let $\phi_{x}^{\prime}$ be the map $B / k \rightarrow A / k$ obtained by factoring multiplication by $p$ on $A$ through $\phi_{x}$. The condition that $h_{\tau}^{\prime}(x)=0$ is equivalent to requiring that the map $\phi_{x}^{\prime}: \operatorname{Lie}(A / k)_{p_{\tau}}^{*} \rightarrow \operatorname{Lie}(B / k)_{p_{\tau}}^{*}$ be zero. This in turn is equivalent to requiring that the kernel of $\phi_{x}^{\prime}$ on $H_{\mathrm{DR}}^{1}(A / k)_{p_{\tau}}$ contain $\operatorname{Lie}(A / k)_{p_{\tau}}^{*}$; as both have dimension one they must be equal. On the other hand, the kernel of $\phi_{x}^{\prime}$ on $H_{\mathrm{DR}}^{1}(A / k)_{p_{\tau}}$ is equal to $\phi_{x}\left(H_{\mathrm{DR}}^{1}(B / k)\right)_{p_{\tau}}$, so $h_{\tau}^{\prime}(x)=0$ if and only if $\phi_{x}\left(H_{\mathrm{DR}}^{1}(B / k)\right)_{p_{\tau}}$ is equal to $\operatorname{Lie}(A / k)_{p_{\tau}}^{*}$ as claimed.

For the second claim, we use Dieudonné theory. Let $\tilde{\mathcal{D}}_{A}$ and $\tilde{\mathcal{D}}_{B}$ denote the contravariant Dieudonné modules of $A\left[p^{\infty}\right]$ and $B\left[p^{\infty}\right]$, respectively. As usual, there is a natural isomorphism $\tilde{\mathcal{D}}_{A} / p \tilde{\mathcal{D}}_{A} \cong H_{\mathrm{DR}}^{1}(A / k)$, and a similar isomorphism for $B$.

The map $\phi_{x}$ induces an injection of $\tilde{\mathcal{D}}_{B}$ into $\tilde{\mathcal{D}}_{A}$, whose image contains $p \tilde{\mathcal{D}}_{A}$; under the above isomorphisms the map $\phi_{x}$ induces on de Rham cohomology is simply the $\bmod p$ reduction of this injection.

Now by the proof of the first part of the proposition (and the symmetry of $\phi_{x}$ and $\left.\phi_{x}^{\prime}\right), h_{\tau}(x)$ is zero if, and only if, $\phi_{x}^{\prime}\left(H_{\mathrm{DR}}^{1}(A / k)_{p_{\tau}}\right)$ is equal to $\operatorname{Lie}(B / k)_{p_{\tau}}^{*}$. In the language of Dieudonné theory, this occurs if and only if $\phi_{x}^{\prime}\left(\tilde{\mathcal{D}}_{A}\right)_{p_{\tau}}$ is equal to $V_{B}\left(\left(\tilde{\mathcal{D}}_{B}\right)_{p_{\sigma \tau}}\right)$. Since $\phi_{x}$ is injective on Dieudonné modules, we can check this equality after composition with $\phi_{x}$; i.e. $h_{\tau}(x)$ is zero if and only if $p\left(\tilde{\mathcal{D}}_{A}\right)_{p_{\tau}}$ equals $\phi_{x}\left(V_{B}\left(\left(\tilde{\mathcal{D}}_{B}\right)_{p_{\sigma \tau}}\right)\right)$ (or, equivalently, if and only if $p\left(\tilde{\mathcal{D}}_{A}\right)_{p_{\tau}}$ equals $\left.V_{A}\left(\phi_{x}\left(\tilde{\mathcal{D}}_{B}\right)_{p_{\sigma \tau}}\right)\right)$. 
Finally, as $V_{A}$ is injective and $V_{A} F_{A}=p$, we have that $h_{\tau}(x)$ is zero if and only if $\phi_{x}\left(\tilde{\mathcal{D}}_{B}\right)_{p_{\sigma \tau}}$ is equal to $F_{A}\left(\left(\tilde{\mathcal{D}}_{A}\right)_{p_{\tau}}\right)$. In terms of de Rham cohomology, this occurs if and only if $\phi_{x}\left(H_{\mathrm{DR}}^{1}(B / k)_{p_{\sigma \tau}}\right)$ is equal to $\operatorname{Fr}\left(H_{\mathrm{DR}}^{1}\left(A^{(p)} / k\right)_{p_{\sigma \tau}}\right)$.

In light of this proposition, we define $Y_{S, S^{\prime}}$ to be the subscheme of $X_{U, p}(\mathcal{V})$ whose $T$-points are tuples $\left(A, \lambda, \rho, B, \lambda^{\prime}, \phi\right)$ that satisfy:

1. $\phi\left(H_{\mathrm{DR}}^{1}(B / T)_{p_{\tau}}\right)=\operatorname{Lie}(A / T)_{p_{\tau}}^{*}$ if $\tau$ is in $S^{\prime}$, and

2. $\phi\left(H_{\mathrm{DR}}^{1}(B / T)_{p_{\tau}}\right)=\operatorname{Fr}\left(H_{\mathrm{DR}}^{1}\left(A^{(p)} / T\right)_{p_{\tau}}\right)$ if $\sigma^{-1} \tau$ is in $S$.

The proposition shows that the underlying point sets of $X_{U, p}(\mathcal{V})_{S, S^{\prime}}$ and $Y_{S, S^{\prime}}$ are the same. Since $X_{U, p}(\mathcal{V})_{S, S^{\prime}}$ is reduced, it follows that we have an isomorphism:

$$
X_{U, p}(\mathcal{V})_{S, S^{\prime}} \cong Y_{S, S^{\prime}}^{\mathrm{red}} .
$$

Now let $Z_{S, S^{\prime}}$ be the scheme representing the functor that takes a $k_{0}$-scheme $T$ to the set of isomorphism classes of tuples $(A, \lambda, \rho, M)$, where $(A, \lambda, \rho)$ is a point of $X_{U}(\mathcal{V})$, and $M$ is an $\mathcal{O}_{F}$-stable subbundle of $H_{\mathrm{DR}}^{1}(A / T)$ that satisfies:

1. $M_{p_{\tau}}$ is locally free of rank one for all $\tau$,

2. $M_{q_{\tau}}=M_{p_{\tau}}^{\perp}$ for all $\tau$,

3. $M_{p_{\tau}}=\operatorname{Lie}(A / T)_{p_{\tau}}^{*}$ if $\tau$ lies in $S^{\prime}$, and

4. $M_{p_{\tau}}=\operatorname{Fr}\left(H_{\mathrm{DR}}^{1}\left(A^{(p)} / T\right)_{p_{\tau}}\right)$ if $\sigma^{-1} \tau$ lies in $S$.

The structure of $Z_{S, S^{\prime}}$ is straightforward to describe explicitly. It is a fiber product of $\mathbb{P}^{1}$-bundles over a closed stratum of $X_{U}(\mathcal{V})$. Let $\Sigma_{S, S^{\prime}}$ be the set of all $\tau$ with $\tau$ in $S^{\prime}$ and $\sigma^{-1} \tau$ in $S$. If $(A, \lambda, \rho, M)$ is a point of $Z_{S, S^{\prime}}$, then $(A, \lambda, \rho)$ lies in the stratum $X_{U}(\mathcal{V})_{\Sigma_{S, S^{\prime}}}$.

In fact, $Z_{S, S^{\prime}}$ is simply a product of $\mathbb{P}^{1}$-bundles over this stratum. Let $\overline{\mathcal{A}}$ be the universal abelian scheme on $X_{U}(\mathcal{V})_{\Sigma_{S, S^{\prime}}}$, and for each $\tau$, let $\overline{\mathcal{E}}_{\tau}$ denote the rank two bundle $H_{\mathrm{DR}}^{1}\left(\overline{\mathcal{A}} / X_{U}(\mathcal{V})_{\Sigma_{S, S^{\prime}}}\right)_{p_{\tau}}$. Let $\Theta_{S, S^{\prime}}$ denote the set of all $\tau$ with $\tau$ not in $S^{\prime}$ and $\sigma^{-1} \tau$ not in $S$. Then $Z_{S, S^{\prime}}$ is simply the fiber product (over $\left.X_{U}(\mathcal{V})_{\Sigma_{S, S^{\prime}}}\right)$ of the bundles $\mathbb{P}\left(\overline{\mathcal{E}}_{\tau}\right)$, for $\tau$ in $\Theta_{S, S^{\prime}}$.

There is an obvious map $Y_{S, S^{\prime}} \rightarrow Z_{S, S^{\prime}}$ that takes $\left(A, \lambda, \rho, B, \lambda^{\prime}, \phi\right)$ to $\left(A, \lambda, \rho, \phi\left(H_{\mathrm{DR}}^{1}(B / T)\right)\right)$.

Proposition 5.7 If $S \cup S^{\prime}$ is all of $\mathbb{S}$, then the map $Y_{S, S^{\prime}} \rightarrow Z_{S, S^{\prime}}$ is a bijection on points.

Proof. We construct an inverse map. Given a $k$-valued point $(A, \lambda, \rho, M)$, of $Z_{S, S^{\prime}}$, with $k$ perfect, we identify $M$ with a subspace of the Dieudonné module $\mathcal{D}_{A}$ of $A[p]$. Note that if $\tau$ is in $S^{\prime}$, then $M_{p_{\tau}}$ is equal to $V_{A}\left(\left(\mathcal{D}_{A}\right)_{p_{\sigma \tau}}\right)$, and if $\sigma^{-1} \tau$ is in $S$, then $M_{p_{\tau}}$ is equal to $F_{A}\left(\left(\mathcal{D}_{A}\right)_{p_{\sigma^{-1}} \tau}\right)$.

For a given $\tau$, either $\sigma^{-1} \tau$ is in $S^{\prime}$ or $\sigma^{-1} \tau$ is in $S$. In the former case $M_{p_{\sigma^{-1} \tau}}$ is equal to $V_{A}\left(\left(\mathcal{D}_{A}\right)_{p_{\tau}}\right)$, and hence clearly contains $V_{A}\left(M_{p_{\tau}}\right)$. In the latter case $M_{p_{\tau}}=F_{A}\left(\left(\mathcal{D}_{A}\right)_{p_{\sigma^{-1}}}\right)$, and hence $V_{A}\left(M_{p_{\tau}}\right)=0$. Either way, we 
have $V_{A}\left(M_{p_{\tau}}\right) \subset M_{p_{\sigma_{-1} 1_{\tau}}}$. It follows that $M$ is stable under $V_{A}$; a similar argument shows it is stable under $F_{A}$.

Thus, exactly as in the proof of Proposition 4.6, we obtain a subgroup $K$ of $A$. Let $B=A / K$, and let $\phi$ be the quotient map $A \rightarrow B$. We have a polarization $\lambda^{\prime}$ on $B=A / K$, such that $p \lambda=\phi^{\vee} \lambda^{\prime} \phi$, and the point $\left(A, \lambda, \rho, B, \lambda^{\prime}, \phi\right)$ is a point of $Y_{S, S^{\prime}}$. This map is easily checked to be an inverse map (at the level of points) to the natural map $Y_{S, S^{\prime}} \rightarrow Z_{S, S^{\prime}}$.

Theorem 5.8 The natural morphism $X_{U, p}(\mathcal{V})_{S, S^{\prime}} \rightarrow Z_{S, S^{\prime}}$ is a Frobenius factor.

Proof. This is immediate from Proposition 4.8 and the above result.

If $S$ and $S^{\prime}$ are disjoint, so that $X_{U, p}(\mathcal{V})_{S, S^{\prime}}$ has codimension zero in the special fiber, then we can go further.

Lemma 5.9 If $S$ and $S^{\prime}$ are disjoint, then $\Sigma_{S, S^{\prime}}$ is sparse.

Proof. If both $\tau$ and $\sigma \tau$ are in $\Sigma_{S, S^{\prime}}$, then $\tau$ must be in both $S$ and $S^{\prime}$.

In this case we can apply the results of the previous section. Let $\mathcal{V}^{\prime}$ be a two-dimensional $F$-vector space with pairing such that $\mathcal{V}^{\prime}\left(\mathbb{A}_{\mathbb{Q}}^{f}\right) \cong \mathcal{V}\left(\mathbb{A}_{\mathbb{Q}}^{f}\right)$ but for which

1. $r_{\tau}\left(\mathcal{V}^{\prime}\right)=2$ if $\tau \in \Sigma_{S, S^{\prime}}$,

2. $r_{\tau}\left(\mathcal{V}^{\prime}\right)=0$ if $\sigma \tau \in \Sigma_{S, S^{\prime}}$, and

3. $r_{\tau}\left(\mathcal{V}^{\prime}\right)=1$ otherwise.

Then, by Theorem 4.9, $X_{U}(\mathcal{V})_{S_{*}\left(S, S^{\prime}\right)}$ is (up to a Frobenius factor) a product of $\mathbb{P}^{1}$-bundles over $X_{U}\left(\mathcal{V}^{\prime}\right)$. Since $Z_{S, S^{\prime}}$ is (up to a Frobenius factor) a product of $\mathbb{P}^{1}$-bundles over $X_{U}(\mathcal{V})_{S_{*}\left(S, S^{\prime}\right)}$, we can express $Z_{S, S^{\prime}}$ as a product of $\mathbb{P}^{1}$-bundles over $X_{U}(\mathcal{V})^{\prime}$. Lemma 4.10, allows us to do this entirely in terms of natural bundles on $X_{U}\left(\mathcal{V}^{\prime}\right)$.

More precisely, let $\overline{\mathcal{A}}^{\prime}$ be the universal abelian variety on $X_{U}\left(\mathcal{V}^{\prime}\right)$, and for each $\tau$ let $\overline{\mathcal{E}}_{\tau}^{\prime}$ be the vector bundle $H_{\mathrm{DR}}^{1}\left(\overline{\mathcal{A}}^{\prime} / X_{U}\left(\mathcal{V}^{\prime}\right)\right)_{p_{\tau}}$. Let $Z_{S, S^{\prime}}^{\prime}$ be the fiber product (over $X_{U}\left(\mathcal{V}^{\prime}\right)$ ) of the bundles $\mathbb{P}\left(\mathcal{E}_{\tau}^{\prime}\right)$, for $\tau$ in $\Sigma_{S, S^{\prime}} \cup \Theta_{S, S^{\prime}}$. By Lemma 4.10 and Theorem 4.9 we obtain a map $Z_{S, S^{\prime}} \rightarrow Z_{S, S^{\prime}}^{\prime}$ that is a Frobenius factor. Composing this with the map $X_{U, p}(\mathcal{V})_{S, S^{\prime}} \rightarrow Z_{S, S^{\prime}}$, we obtain:

Theorem 5.10 The map $X_{U, p}(\mathcal{V})_{S, S^{\prime}} \rightarrow Z_{S, S^{\prime}}^{\prime}$ described above is a Frobenius factor.

This gives a complete global description of the irreducible components of $X_{U, p}(\mathcal{V})$ in terms of Shimura varieties for various inner forms of $G$.

Example 5.11 Suppose $F^{+}$is real quadratic, and let $\tau_{1}$ and $\tau_{2}$ denote its two real embeddings. Suppose further that $p$ is inert in $F^{+}$. In this setting, the framework we have established divides $X_{U, p}(\mathcal{V})$ into four pieces: 
1. $S=\left\{\tau_{1}, \tau_{2}\right\} ; S^{\prime}$ is empty. Here $\Sigma_{S, S^{\prime}}$ and $\Theta_{S, S^{\prime}}$ are both empty, and thus the scheme $Z_{S, S^{\prime}}$ is isomorphic to $X_{U}(\mathcal{V})$. In particular $X_{U, p}(\mathcal{V})_{S, S^{\prime}}$ differs from $X_{U}(\mathcal{V})$ by at most a Frobenius factor. In fact, it is easy to see that in this case the two are isomorphic, as $X_{U, p}(\mathcal{V})_{S, S^{\prime}}$ is the image of $X_{U}(\mathcal{V})_{k_{0}}$ under the map that associates the relative Frobenius to every abelian variety parametrized by $X_{U}(\mathcal{V})$.

2. $S$ is empty, $S^{\prime}=\left\{\tau_{1}, \tau_{2}\right\}$. Again $\Sigma_{S, S^{\prime}}$ and $\Theta_{S, S^{\prime}}$ are empty, and the scheme $Z_{S, S^{\prime}}$ is isomorphic to $X_{U}(\mathcal{V})$. Here $X_{U, p}(\mathcal{V})_{S, S^{\prime}}$ is the image of $X_{U}(\mathcal{V})_{k_{0}}$ under the map that associates the Verschiebung morphism to every abelian variety parametrized by $X_{U}(\mathcal{V})$.

3. $S=\left\{\tau_{1}\right\}, S^{\prime}=\left\{\tau_{2}\right\}$. Here $\Sigma_{S, S^{\prime}}=\left\{\tau_{2}\right\}$, and $\Theta_{S, S^{\prime}}=\left\{\tau_{1}\right\}$. The scheme $Z_{S, S^{\prime}}^{\prime}$ is a $\mathbb{P}^{1} \times \mathbb{P}^{1}$-bundle over a zero-dimensional Shimura variety, or, more prosaically, a disjoint union of copies of $\mathbb{P}^{1} \times \mathbb{P}^{1}$ 's.

4. $S=\left\{\tau_{2}\right\}, S^{\prime}=\left\{\tau_{1}\right\}$ exhibits the same behavior as the previous case, with the roles of $\tau_{1}$ and $\tau_{2}$ reversed.

It is worth noting that this description is exactly analogous to Helmuth Stamm's description of the mod $p$ reduction of a Hilbert modular surface with $\Gamma_{0}(p)$ level structure over a real quadratic field in which $p$ is inert $\mathrm{St}$.

Example 5.12 If in the previous example we had chosen to consider a prime $p$ that was split in $F^{+}$, the behavior for $S=\left\{\tau_{1}, \tau_{2}\right\}$ and $S$ empty would remain the same, but when $S=\left\{\tau_{1}\right\}$ and $S^{\prime}=\left\{\tau_{2}\right\}$, we would have $\Theta_{S, S^{\prime}}$ and $\Sigma_{S, S^{\prime}}$ both empty. Thus $Z_{S, S^{\prime}}$ is isomorphic to $X_{U}(\mathcal{V})$ in this case as well, and no nontrivial bundles occur.

\section{A semistable model}

We now use Theorem 5.8 to describe a semistable model for $X_{U}(\mathcal{V})$. To do so it suffices to give a semistable model for $\mathcal{M}$. Note that if $x=\mathcal{M}_{\mathbb{S}, \mathbb{S}}$ is the "most singular point" of $\mathcal{M}$, then the completion $\mathcal{M}_{x}$ is given by

$$
\mathcal{M}_{x}=W\left(\overline{\mathbb{F}}_{p}\right)\left[\left[X_{1}, Y_{1}, \ldots, X_{d}, Y_{d}\right]\right] /\left\langle X_{1} Y_{1}-p, \ldots X_{d} Y_{d}-p\right\rangle .
$$

It is well-known how to resolve such a singularity, and its resolution has a particularly nice description in terms of toric geometry.

Note first that it suffices to give a semistable model over $\mathbb{Z}[t]$ for the scheme

$$
\mathcal{M}^{\prime}=\operatorname{Spec} \mathbb{Z}[t]\left[X_{1}, Y_{1}, \ldots, X_{d}, Y_{d}\right] /<X_{1} Y_{1}-t, X_{d} Y_{d}-t>,
$$

as we can then obtain a semistable model for $\mathcal{M}_{x}$ by base change.

The scheme $\mathcal{M}^{\prime}$ can be viewed as a toric variety over $\mathbb{Z}$; it is the $d$-fold fiber product over Spec $\mathbb{Z}[t]$ of the scheme

$$
\operatorname{Spec} \mathbb{Z}[t][X, Y] /<X Y-t>.
$$


As an affine toric variety (over $\mathbb{Z}$ ), Spec $\mathbb{Z}[t][X, Y] /\langle X Y-t\rangle$ corresponds to the cone $C$ in $\mathbb{R}^{2}$ spanned by $(1,0)$ and $(1,1)$; the morphism to $\operatorname{Spec} \mathbb{Z}[t]$ corresponds to the map $C \rightarrow \mathbb{R}_{\geq 0}$ given by projection onto the first coordinate. As $\mathcal{M}^{\prime}$ is the $d$-fold fiber product of this toric variety over Spec $\mathbb{Z}[t]$, it is the affine toric variety whose cone is the $d$-fold fiber product $C_{d}$ of $C$ over $\mathbb{R}_{\geq 0}$. More prosaically, $C_{d}$ is the cone in $\mathbb{R}^{d+1}$ over the $n$-cube

$$
Q=\{1\} \times[0,1] \times \cdots \times[0,1] .
$$

Fulton ( $[\mathrm{Fu}, 2.6)$ shows how to resolve the singularities of a toric variety by giving a refinement of its fan whose cones are simplicial and unimodular. In this case this amounts to fixing a decomposition $\mathcal{C}$ of $Q$ into unimodular simplices, any two of which intersect in a common face of both. The cones over these simplices are then a fan $\mathcal{F}$ consisting of unimodular cones that refines $C_{d}$. To fix ideas, we let $\mathcal{C}$ be the decomposition whose top dimensional simplices are indexed by elements of the symmetric group $S_{d}$, and for a given $\sigma \in S_{d}$ are given by

$$
C_{\sigma}=\left\{\left(1, x_{1}, \ldots, x_{d}\right) \in Q: x_{\sigma(1)} \leq x_{\sigma(2)} \leq \cdots \leq x_{\sigma(d)}\right\} .
$$

Let $\left(\mathcal{M}^{\prime}\right)^{\text {ss }}$ be the toric variety (over $\mathbb{Z}$ ) associated to $\mathcal{F}$. The map of fans $\mathcal{F} \rightarrow C_{d}$ induces a birational morphism from $\left(\mathcal{M}^{\prime}\right)^{\text {ss }}$ to $\mathcal{M}^{\prime}$. As all the cones in $\mathcal{F}$, the boundary of $\left(\mathcal{M}^{\prime}\right)^{\text {ss }}$ are unimodular, its boundary is a divisor with normal crossings. In particular the fiber over $t=0$ is a divisor with normal crossings.

After a base change from $\mathbb{Z}[t]$ to $W\left(\overline{\mathbb{F}}_{p}\right)$, we arrive at a semistable resolution $\mathcal{M}_{x}^{\text {ss }}$ of $\mathcal{M}_{x}$. The strata of the special fiber of $\mathcal{M}_{x}^{\text {ss }}$ have a simple description in terms of the combinatorics of $\mathcal{C}$. In particular, irreducible components of the special fiber correspond to 0 -simplices in $\mathcal{C}$ (i.e., vertices of $Q$ ). A collection of irreducible components intersect nontrivially if their corresponding vertices form the vertices of a simplex in $\mathcal{C}$. For an $r$-simplex $P$ in $\mathcal{C}$, let $\mathcal{M}_{P}^{\text {ss }}$ be the intersection of the irreducible components of the special fiber of $\mathcal{M}_{x}^{\text {ss }}$ that correspond to the vertices of $P$.

The image of $\mathcal{M}_{P}^{\text {ss }}$ in $\mathcal{M}$ is determined by the smallest face of $Q$ containing $P$. More precisely, fix a bijection $i \mapsto \tau_{i}$ of $\{1, \ldots, d\}$ with the set of real embeddings of $F^{+}$, and define:

$$
\begin{aligned}
& S_{P}=\left\{\tau_{i}: \exists\left(1, x_{1}, \ldots, x_{d}\right) \in P, x_{i}>0\right\} \\
& S_{P}^{\prime}=\left\{\tau_{i}: \exists\left(1, x_{1}, \ldots, x_{d}\right) \in P, x_{i}<1\right\} .
\end{aligned}
$$

Then the image of $\mathcal{M}_{P}^{\text {ss }}$ in $\mathcal{M}_{x}$ is the intersection of $\mathcal{M}_{x}$ with $\mathcal{M}_{S_{P}, S_{P}^{\prime}}$. In particular, if $P$ is a top-dimensional simplex, then $\mathcal{M}_{P}^{\text {ss }}$ is a point lying over $x$. Similarly, if $P$ is a codimension 1 simplex, then $\mathcal{M}_{P}^{\text {ss }}$ is a rational curve; it lies over $x$ if, and only if, $P$ is not contained in a proper face of $Q$.

The semistable resolution of $\mathcal{M}_{x}$ gives rise to a semistable resolution $X_{U}(\mathcal{V})^{\text {ss }}$ of $X_{U}(\mathcal{V})$, and the combinatorics of $\mathcal{M}^{s s}$ reflect the combinatorics of $X_{U}(\mathcal{V})^{\text {ss }}$. In particular each vertex $V$ of $Q$ corresponds to the irreducible component of 
the special fiber of $\mathcal{M}^{\text {ss }}$ that dominates $\mathcal{M}_{S_{V}, S_{V}^{\prime}}$. For each such $V$, let $X_{U}(\mathcal{V})_{V}^{\text {ss }}$ denote the union of irreducible components of the special fiber of $X_{U}(\mathcal{V})^{\text {ss }}$ that dominate some component of $X_{U}(\mathcal{V})^{\text {ss }}$. This is a disjoint union of smooth varieties over $k_{0}$. Extend this to simplices $P$ of arbitrary dimension in $\mathcal{C}$ by taking $X_{U}(\mathcal{V})_{P}^{\text {ss }}$ to be the intersection of $X_{U}(\mathcal{V})_{V}^{\text {ss }}$ over all vertices $V$ of $P$.

For $0 \leq r \leq d$, let $X_{U}(\mathcal{V})^{\mathrm{ss}},(r)$ denote the disjoint union of the intersections of $r+1$ distinct irreducible components of the special fiber of $X_{U}(\mathcal{V})^{\mathrm{ss}}$. As $X_{U}(\mathcal{V})^{\text {ss }}$ is semistable, these are all smooth varieties over $\overline{\mathbb{F}}_{p}$.

In codimensions $d$ and $d-1$ these spaces are particularly easy to desribe. In particular $X_{U}(\mathcal{V})^{\mathrm{ss},}(d)$ is simply the disjoint union over top-dimensional simplices $P$ in $\mathbb{Q}$ of $X_{U}(\mathcal{V})_{P}^{\text {ss }}$, and each of these is simply a copy of $X_{U}(\mathcal{V})_{\mathbb{S}, \mathbb{S}}$.

Similarly, $X_{U}(\mathcal{V})^{\text {ss },(d-1)}$ is the disjoint union of $X_{U}(\mathcal{V})_{P}^{\text {ss }}$, for $P$ a $d$-1-simplex of $\mathcal{C}$. We have:

1. If $P$ is contained in the relative interior of $Q$, then $X_{U}(\mathcal{V})_{P}^{\text {ss }}$ is a $\mathbb{P}^{1}$-bundle over the discrete set $X_{U}(\mathcal{V})_{\mathbb{S}, \mathbb{S}}$.

2. If $P$ is contained in a face $x_{i}=0$ of $Q$, then $X_{U}(\mathcal{V})_{P}^{\text {ss }}$ maps isomorphically

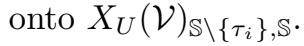

3. If $P$ is contained in a face $x_{i}=1$ of $Q$, then $X_{U}(\mathcal{V})_{P}^{\text {ss }}$ maps isomorphically onto $X_{U}(\mathcal{V})_{\mathbb{S}, \mathbb{S} \backslash\left\{\tau_{i}\right\}}$.

If we fix orientations on each simplex in $\mathcal{C}$, we get a map

$$
H_{\text {et }}^{0}\left(X_{U}(\mathcal{V})_{\overline{\mathbb{F}}_{p}^{\mathrm{ss}},(d-1)}, \mathbb{Q}_{l}\right) \rightarrow H_{\text {et }}^{0}\left(X_{U}(\mathcal{V})_{\overline{\mathbb{F}}_{p}}^{\mathrm{ss},(d)}, \mathbb{Q}_{l}\right)
$$

defined as follows: For each $d$-dimensional simplex $P$, and each face $P^{\prime}$ of $P$, the map

$$
H_{\text {ét }}^{0}\left(X_{U}(\mathcal{V})_{P^{\prime}}^{\text {ss }}, \mathbb{Q}_{l}\right) \rightarrow H_{\text {ét }}^{0}\left(X_{U}(C V)_{P}^{\mathrm{ss}}, \mathbb{Q}_{l}\right)
$$

is the canonical map on cohomology if the chosen orientation for $P^{\prime}$ agrees with the orientation $P^{\prime}$ acquires from $P$, and is the negative of this map otherwise.

We can interpret the cokernel of this map purely in terms of $X_{U}(\mathcal{V})$, rather than its resolution. In partiulcar let $X_{U}(\mathcal{V})^{(d-1)}$ be the disjoint union of the 1-strata in the special fiber of $X_{U}(\mathcal{V})$; that is, the disjoint union of the strata $X_{U}(\mathcal{V})_{\mathbb{S}, \mathbb{S} \backslash \tau}$ and $X_{U}(\mathcal{V})_{\mathbb{S} \backslash \tau, \mathbb{S}}$ for all $\tau$.

We then have a commutative diagram:

$$
\begin{aligned}
& H_{\text {ét }}^{0}\left(X_{U}(\mathcal{V})_{\mathbb{\mathbb { F }}_{p}}^{(d-1)}, \mathbb{Q}_{l}\right) \quad \rightarrow \quad H_{\text {ét }}^{0}\left(\left(X_{U}(\mathcal{V})_{\mathbb{S}, \mathbb{S}}\right)_{\mathbb{F}_{p}}, \mathbb{Q}_{l}\right)
\end{aligned}
$$

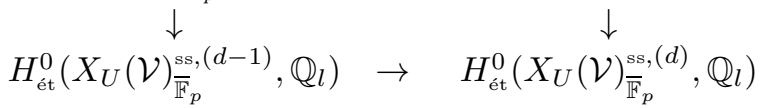

where the vertical arrows are given by pullback.

An easy computation then shows:

Proposition 6.1 The vertical maps in the above diagram are a quasiisomorphism. In particular the cokernel of the top horizontal map is isomorphic to the cokernel of the bottom horizontal map. 


\section{Cohomology}

We now illustrate how the geometric results of the previous sections, together with the weight spectral sequence of Rapoport-Zink, can be used to obtain a Jacquet-Langlands correspondence on the level of étale cohomology. The technical complications involved in doing this in general are beyond the scope of this paper, and will be addressed in future work. Here, we will content ourselves with considering the case where $p$ is inert in $F^{+}$, and $d$ is even.

Our techniques require as input Shimura varieties that are proper. It will therefore be necessary to work not with the moduli spaces $X_{U}(\mathcal{V})$, but with the more general $X_{U}^{D}(\mathcal{V})$ where $D$ is a quaternion algebra. We thus depart from the convention, in force in the rest of the paper, of considering only the former.

Fix a 4-dimensional central simple $F$-algebra $D$ split at $p$, and a rank one left $D$-module $\mathcal{V}$ equipped with a pairing such that $r_{\tau}(\mathcal{V})=1$ for all $\tau \in \mathbb{S}$. Consider the Shimura variety $X=X_{U}^{D}(\mathcal{V})$ for some suitable $U$. Our first objective will be to understand the finite set $X_{\mathbb{S}}\left(\overline{\mathbb{F}}_{p}\right)$.

Choose disjoint subsets $S$ and $S^{\prime}$ of $\mathbb{S}$, such that $S=\sigma S^{\prime}$. (Since $p$ is inert in $F^{+}$, there is a unique way to do this up to interchanging $S$ and $S^{\prime}$.

Fix a $\mathcal{V}^{\prime}$ isomorphic to $\mathcal{V}$ at all finite places with $r_{\tau}\left(\mathcal{V}^{\prime}\right)=2$ for $\tau \in S$ and $r_{\tau}\left(\mathcal{V}^{\prime}\right)=0$ for $\tau \in S^{\prime}$. Thus defines a unitary group $G^{\prime}$ isomorphic to $G$ at all non-archimedean places; let $U^{\prime}$ be the subgroup of $G^{\prime}\left(\mathbb{A}_{Q}\right)$ corresponding to $U$. Let $X^{\prime}=X_{U^{\prime}}^{D}\left(\mathcal{V}^{\prime}\right)$; it is a zero-dimensional unitary Shimura variety. We fix an identification of $\mathcal{V}^{\prime}$ with $\mathcal{V}$ at all finite places.

The inclusion of $X_{\mathbb{S}}$ in $X_{S}$, followed by the map $X_{S} \rightarrow X^{\prime}$ constructed in section 4, yields a map

$$
\alpha_{1}: X_{\mathbb{S}}\left(\overline{\mathbb{F}}_{p}\right) \rightarrow X^{\prime}\left(\overline{\mathbb{F}}_{p}\right) .
$$

We can construct another map from $X_{\mathbb{S}}\left(\overline{\mathbb{F}}_{p}\right)$ to $X^{\prime}\left(\overline{\mathbb{F}}_{p}\right)$ as follows. Fix a $\mathcal{V}^{\prime \prime}$ isomorphic to $\mathcal{V}$ at all finite places with $r_{\tau}\left(\mathcal{V}^{\prime \prime}\right)=0$ for $\tau \in S$ and $r_{\tau}\left(\mathcal{V}^{\prime \prime}\right)=2$ for $\tau \in S^{\prime}$. If we let $X^{\prime \prime}$ denote $X_{U^{\prime \prime}}\left(\mathcal{V}^{\prime \prime}\right)$, and fix an identification of $\mathcal{V}^{\prime \prime}$ with $\mathcal{V}$ at all finite places, then we obtain (as in section 4) a map from $X_{S^{\prime}}$ to $X^{\prime \prime}$. On the other hand, given $\left(A^{\prime \prime}, \lambda^{\prime \prime}, \rho^{\prime \prime}\right) \in X^{\prime \prime}\left(\overline{\mathbb{F}}_{p}\right)$, the $U^{\prime \prime}$-level structure $\rho^{\prime \prime}$ on $A^{\prime \prime}$ induces a $U^{\prime}$-level structure on $A^{\prime \prime}$ (as we have fixed identifications of $\mathcal{V}$ with $\mathcal{V}^{\prime}$ and $\left.\mathcal{V}^{\prime \prime}\right)$, and hence also a $U^{\prime}$-level structure $\rho^{\prime}$ on $\left(A^{\prime \prime}\right)^{(p)}$. Then $\left(\left(A^{\prime \prime}\right)^{(p)},\left(\lambda^{\prime \prime}\right)^{(p)}, \rho^{\prime}\right)$ is a point in $X^{\prime}\left(\overline{\mathbb{F}}_{p}\right)$.

Composing the inclusion of $X_{\mathbb{S}}$ into $X_{S^{\prime}}$ with the map $X_{S^{\prime}} \rightarrow X^{\prime \prime}$, and then applying the above construction gives a map

$$
\alpha_{2}: X_{\mathbb{S}}\left(\overline{\mathbb{F}}_{p}\right) \rightarrow X^{\prime}\left(\overline{\mathbb{F}}_{p}\right) .
$$

Knowing $\alpha_{1}(x)$ for some $x \in X_{\mathbb{S}}\left(\overline{\mathbb{F}}_{p}\right)$ is insufficient to recover $x$. The "missing data" turns out to be that of a $\Gamma_{0}(p)$-level structure on $\alpha_{1}(x)$. Let us make this more precise.

Let $x=(A, \lambda, \rho)$ be a point in $X_{\mathbb{S}}\left(\overline{\mathbb{F}}_{p}\right)$, and let $\left(A^{\prime}, \lambda^{\prime}, \rho^{\prime}\right)$ be the tuple corresponding to $\alpha_{1}(x) \in X^{\prime}\left(\overline{\mathbb{F}}_{p}\right)$. The construction in section 4 gives us an isogeny $\pi_{1}: A \rightarrow A^{\prime}$ whose kernel is contained in $A[p]$. This map induces a 
map $\mathcal{D}_{A^{\prime}} \rightarrow \mathcal{D}_{A}$, where $\mathcal{D}_{A}$ and $\mathcal{D}_{A^{\prime}}$ are the contravariant Dieudonné modules of $A[p]$ and $A^{\prime}[p]$ respectively. Define:

$$
M_{A} \subset \bigoplus_{\tau}\left(\mathcal{D}_{A}\right)_{p_{\tau}}
$$

as follows:

1. $\left(M_{A}\right)_{p_{\tau}}=0$ for $\tau \in S$, and

2. $\left(M_{A}\right)_{p_{\tau}}=V_{A}\left(\left(\mathcal{D}_{A}\right)_{p_{\sigma \tau}}\right)=F_{A}\left(\left(\mathcal{D}_{A}\right)_{p_{\sigma-1}{ }_{\tau}}\right)$ for $\tau \in S^{\prime}$.

Note that $M_{A}$ is stable under $F_{A}$ and $V_{A}$.

Let $M_{A^{\prime}}$ denote the preimage of $M_{A}$ under the map

$$
\pi_{1}: \bigoplus_{\tau}\left(\mathcal{D}_{A^{\prime}}\right)_{p_{\tau}} \rightarrow \bigoplus_{\tau}\left(\mathcal{D}_{A}\right)_{p_{\tau}}
$$

As with $M_{A}, M_{A^{\prime}}$ is stable under $F_{A^{\prime}}$ and $V_{A^{\prime}}$. Note that because the kernel $L$ of $\pi_{1}: \mathcal{D}_{A^{\prime}} \rightarrow \mathcal{D}_{A}$ satisfies $\operatorname{dim} L_{p_{\tau}}=2$ for $\tau \in S$ and $L_{p_{\tau_{2}}}=0$ for $\tau \in S^{\prime}$, we have $\operatorname{dim}\left(M_{A^{\prime}}\right)_{p_{\tau}}=2$ for all $\tau$ in $\mathbb{S}$. (The dimensions here are 2 instead of 1 because we are working with the moduli space $X_{U}^{D}(\mathcal{V})$ rather than the simpler moduli problem $X_{U}(\mathcal{V})$.)

Then the subspace $M$ of $\left(\mathcal{D}_{A^{\prime}}\right)$ defined by $M_{p_{\tau}}=\left(M_{A^{\prime}}\right)_{p_{\tau}}$ and $M_{q_{\tau}}=M_{p_{\tau}}^{\perp}$ for $\tau \in \mathbb{S}$ is also stable under $F_{A^{\prime}}$ and $V_{A^{\prime}}$, and is a free $\mathcal{O}_{F} / p$-module of rank 2 , stable under the action of $\mathcal{O}_{D} / p$. This module sits in an exact sequence

$$
0 \rightarrow M \rightarrow \mathcal{D}_{A^{\prime}} \rightarrow \mathcal{D}\left(K_{M}\right) \rightarrow 0,
$$

where $K_{M} \subset A^{\prime}[p]$ is a maximal isotropic subgroup of $A^{\prime}[p]$ and $\mathcal{D}\left(K_{M}\right)$ is its Dieudonné module.

Let $B=A^{\prime} / K_{M}$, and let $\phi$ be the natural quotient map. Since $K_{M}$ is maximal isotropic, there is a unique prime-to- $p$ polarization $\lambda_{B}$ on $B$ such that $p \lambda^{\prime}=\phi^{\vee} \lambda_{B} \phi$. The cokernel of $\phi: \mathcal{D}_{B} \rightarrow \mathcal{D}_{A^{\prime}}$ is simply $\mathcal{D}_{A^{\prime}} / M$, and is thus a locally free $\mathcal{O}_{F} \otimes \overline{\mathbb{F}}_{p}$-module of rank one. It follows that $\left(A^{\prime}, \lambda^{\prime}, \rho^{\prime}, B, \lambda_{B}, \phi\right)$ is an $\overline{\mathbb{F}}_{p}$ point on the zero-dimensional variety $X_{U^{\prime}, p}^{D}\left(\mathcal{V}^{\prime}\right)$. We denote this variety by $X_{p}^{\prime}$ in what follows.

Proposition 7.1 The map $X_{\mathbb{S}}\left(\overline{\mathbb{F}}_{p}\right) \rightarrow X_{p}^{\prime}\left(\overline{\mathbb{F}}_{p}\right)$ that associates to $(A, \lambda, \rho)$ the tuple $\left(A^{\prime}, \lambda^{\prime}, \rho^{\prime}, B, \lambda_{B}, \phi\right)$ constructed above is a bijection. Moreover, under this identification of $X_{\mathbb{S}}\left(\overline{\mathbb{F}}_{p}\right)$ with $X_{p}^{\prime}\left(\overline{\mathbb{F}}_{p}\right)$, the maps

$$
\alpha_{1}, \alpha_{2}: X_{\mathbb{S}} \rightarrow X^{\prime}
$$

are identified with the degeneracy maps $X_{p}^{\prime} \rightarrow X^{\prime}$ that "forget" and "mod out by" the $\Gamma_{0}(p)$ level structure, respectively.

Proof. We provide an inverse to the above construction. Let $\left(A^{\prime}, \lambda^{\prime}, \rho^{\prime}, B, \lambda_{B}, \phi\right)$ be an $\overline{\mathbb{F}}_{p}$-point of $X_{p}^{\prime}$. We define a subspace $L$ of $\mathcal{D}_{A^{\prime}}$ by: 
1. $L_{p_{\tau}}=\phi\left(\mathcal{D}_{B}\right)_{p_{\tau}}$ for $\tau \in S$,

2. $L_{p_{\tau}}=0$ for $\tau \in S^{\prime}$, and

3. $L_{q_{\tau}}=L_{p_{\tau}}^{\perp}$ for all $\tau \in \mathbb{S}$.

Since $F$ and $V$ are both zero map on $\left(\mathcal{D}_{A^{\prime}}\right)_{p_{\tau}}$ for $\tau \in S, L$ is a Dieudonné submodule of $\mathcal{D}_{A^{\prime}}$. Note that if $\left(A^{\prime}, \lambda^{\prime}, \rho^{\prime}, B, \lambda_{B}, \phi\right)$ arose from an $(A, \lambda, \rho)$ in $X_{\left\{\tau_{1}, \tau_{2}\right\}}$ via the above construction, then $L$ is simply the kernel of the map $\mathcal{D}_{A^{\prime}} \rightarrow \mathcal{D}_{A}$ induced by the map $A \rightarrow A^{\prime}$ occurring in that construction.

As usual, this module yields an exact sequence

$$
0 \rightarrow L \rightarrow \mathcal{D}_{A^{\prime}} \rightarrow \mathcal{D}\left(K_{L}\right) \rightarrow 0
$$

for some maximal isotropic subgroup $K_{L}$ of $A^{\prime}[p]$. Let $A=A^{\prime} / K_{L}$. The polarization $\lambda^{\prime}$ on $A^{\prime}$ induces a prime-to- $p$ polarization $\lambda$ on $A$, and the $U^{\prime}$-level structure $\rho^{\prime}$ induces a $U$-level structure $\rho$ on $A$. Then $\left(A, \lambda, \frac{1}{\rho} \rho\right)$ is a point on $X$. Moreover, it is clear from the previous paragraph that if $\left(A^{\prime}, \lambda^{\prime}, \rho^{\prime}, B, \lambda_{B}, \phi\right)$ originally arose from an $\overline{\mathbb{F}}_{p}$-point $x$ of $X_{\mathbb{S}}$ via the first construction, then we have $x=\left(A, \lambda, \frac{1}{p} \rho\right)$.

We now verify that the point $\left(A, \lambda, \frac{1}{p} \rho\right)$ we have constructed lies on $X_{\mathbb{S}}$. Consider the Dieudonné modules $\tilde{\mathcal{D}}_{A}, \tilde{\mathcal{D}}_{B}$, and $\tilde{\mathcal{D}}_{A^{\prime}}$ of $A\left[p^{\infty}\right], B\left[p^{\infty}\right]$, and $A^{\prime}\left[p^{\infty}\right]$ respectively. Let $\varphi$ denote the quotient map $A^{\prime} \rightarrow A$.

The map $\varphi$ identifies $\tilde{\mathcal{D}}_{A}$ with a submodule of $\tilde{\mathcal{D}}_{A^{\prime}}$, and in particular identifies $\left(\tilde{\mathcal{D}}_{A}\right)_{p_{\tau}}$ with $p\left(\tilde{\mathcal{D}}_{A^{\prime}}\right)_{p_{\tau}}$ for $\tau \in S^{\prime}$. For $\tau \in S$, the maps $F_{A^{\prime}}$ and $V_{A^{\prime}}$ map $\left(\tilde{\mathcal{D}}_{A^{\prime}}\right)_{p_{\sigma^{-1} \tau}}$ and $\left(\tilde{\mathcal{D}}_{A^{\prime}}\right)_{p_{\sigma \tau}}$ isomorphically onto $\left(\tilde{\mathcal{D}}_{A^{\prime}}\right)_{p_{\tau}}$, and so map $\left(\tilde{\mathcal{D}}_{A}\right)_{p_{\sigma^{-1}} \tau_{\tau}}$ and $\left(\tilde{\mathcal{D}}_{A}\right)_{P_{\sigma \tau}}$ isomorphically onto $p\left(\tilde{\mathcal{D}}_{A^{\prime}}\right)_{p_{\tau}}$. In particular $F_{A^{\prime}}\left(\tilde{\mathcal{D}}_{A}\right)_{p_{\sigma-1}}=$ $V_{A^{\prime}}\left(\tilde{\mathcal{D}}_{A}\right)_{p_{\sigma \tau}}$ and so $\left(A, \lambda, \frac{1}{p} \rho\right)$ lies in $X_{S}$.

On the other hand, by our construction of $A$ we have $\phi\left(\tilde{\mathcal{D}}_{B}\right)_{p_{\tau}}=\varphi\left(\tilde{\mathcal{D}}_{A}\right)_{p_{\tau}}$ for $\tau \in S$. But $F_{B}$ and $V_{B} \operatorname{map}\left(\tilde{\mathcal{D}}_{B}\right)_{p_{\sigma-1_{\tau}}}$ and $\left(\tilde{\mathcal{D}}_{B}\right)_{p_{\sigma \tau}}$ (respectively) isomorphically onto $\left(\tilde{\mathcal{D}}_{B}\right)_{p_{\tau}}$.

We therefore have:

$$
F_{A^{\prime}} \phi\left(\tilde{\mathcal{D}}_{B}\right)_{p_{\tau}}=F_{A^{\prime}} \phi\left(V_{B}\left(\tilde{\mathcal{D}}_{B}\right)_{p_{\sigma \tau}}=F_{A^{\prime}} V_{A^{\prime}} \phi\left(\tilde{\mathcal{D}}_{B}\right)_{p_{\sigma \tau}}=p \phi\left(\tilde{\mathcal{D}}_{B}\right)_{p_{\sigma \tau}} .\right.
$$

Since $\sigma^{2} \tau$ is also in $S$, we similarly have

$$
V_{A^{\prime}} \phi\left(\tilde{\mathcal{D}}_{B}\right)_{p_{\sigma^{2} \tau}}=p \phi\left(\tilde{\mathcal{D}}_{B}\right)_{p_{\sigma \tau}} .
$$

It follows that $F_{A}\left(\tilde{\mathcal{D}}_{A}\right)_{p_{\tau}}=V_{A}\left(\tilde{\mathcal{D}}_{A}\right)_{p_{\sigma^{2} \tau}}$, for all $\tau \in S$, so $\left(A, \lambda, \frac{1}{\rho} \rho\right)$ lies in $X_{S^{\prime}}$.

One now verifies easily that the construction given above is both a left and right inverse to the map $X_{\mathbb{S}}\left(\overline{\mathbb{F}}_{p}\right) \rightarrow X_{p}^{\prime}\left(\overline{\mathbb{F}}_{p}\right)$.

A straightforward calculation shows that under this identification, the maps $\alpha_{1}, \alpha_{2}$ from $X_{\mathbb{S}}$ to $X^{\prime}$ correspond exactly to the two degeneracy maps $X_{p}^{\prime} \rightarrow X^{\prime}$ that "forget the level structure at $p$ " and "mod out by the level structure at $p$ " respectively. 
Remark 7.2 Although we will make no use of the fact here, it is not difficult to generalize this construction to a description of the dimension zero locus for any totally real field $F^{+}$in which every prime of $F^{+}$over $p$ has even residue class degree. When primes of odd residue class degree occur over $p$, the construction here breaks down completely. It is likely that in this case one needs to consider an auxiliary quaternion algebra ramified at the primes of odd residue class degree over $p$. Even if one does this, it is not clear at all what archimedean invariants $r_{\tau}^{\prime}, s_{\tau}^{\prime}$ should be taken.

We now turn to arithmetic questions. The key tool here will be the weight spectral sequence of Rapoport-Zink [RZ2, [Sa. This applies only to proper schemes with semistable reduction. In order to obtain a proper scheme to apply it on, we will need to make the assumption that $D$ is ramified at at least one prime- that is, $D$ is a quaternion algebra rather than a matrix algebra.

In this case, the scheme $X_{U, p}^{D}(\mathcal{V})$ is proper over $W\left(k_{0}\right)$, and has the semistable resolution $X^{\text {ss }}$ described in section 6 . For each $r$, with $0 \leq r \leq d$, let $Y^{(r)}$ be the scheme over $\overline{\mathbb{F}}_{p}$ consisting of the disjoint union of all nonempty $r+1$-fold intersections of irreducible components of the special fiber of $X^{\text {ss }}$.

Fix a prime $l$ different from $p$. The weight spectral sequence (c.f. Sa], Corollary 2.2.4) is a spectral sequence

$$
E_{1}^{p, q}=\bigoplus_{i \geq \max (0,-p)} H_{\mathrm{e} \mathrm{t}}^{q-2 i}\left(Y^{(p+2 i)}, \mathbb{Q}_{l}(-i)\right) \Rightarrow H_{\mathrm{èt}}^{p+q}\left(X_{\bar{K}}^{\mathrm{ss}}, \mathbb{Q}_{l}\right) .
$$

This spectral sequence degenerates at $E_{2}$. In our situation, its nonzero terms are concentrated between $p=-d$ and $p=d$. Thus the sequence

$$
E_{2}^{d, 0}, E_{2}^{d-1,1}, \ldots E_{2}^{1-d, 2 d-1}, E_{2}^{-d, 2 d}
$$

is the sequence of successive quotients of a $2 d+1$-step filtration (the "weight filtration") on $H_{\text {et }}^{d}\left(X_{\overline{\mathrm{ss}}}^{\mathrm{s}}, \mathbb{Q}_{l}\right)$. (Equivalently, this is a filtration of $H_{\mathrm{et}}^{d}\left(X_{U, p}^{D}(\mathcal{V})_{\bar{K}}\right)$, as the blow-up we performed altered only the special fiber.)

We will compute the top and bottom quotients of this filtration. First we need some notation: Let $U_{p}^{\prime}$ be the subgroup of $G^{\prime}\left(\mathbb{A}_{\mathbb{Q}}\right)$ equal to $U^{\prime}$ at all prime to $p$ places but isomorphic to $\Gamma_{0}(p)$ at $p$. Let $S_{2}\left(G^{\prime}, U_{p}^{\prime}, \mathbb{Q}_{l}\right)$ denote the space of algebraic modular forms Gross on $G^{\prime}$ of level $U_{p}^{\prime}$ and "weight 2"; that is, the space of $\mathbb{Q}_{l}$-valued functions on the double coset space $G^{\prime}(\mathbb{Q}) \backslash G^{\prime}\left(\mathbb{A}_{\mathbb{Q}}\right) / U_{p}^{\prime}$. There are two natural degeneracy maps

$$
S_{2}\left(G^{\prime}, U^{\prime}, \mathbb{Q}_{l}\right) \rightarrow S_{2}\left(G^{\prime}, U_{p}^{\prime}, \mathbb{Q}_{l}\right) ;
$$

let $S_{2}\left(G^{\prime}, U_{p}^{\prime}, \mathbb{Q}_{l}\right)^{p \text {-new }}$ denote the " $p$-new" quotient of $S_{2}\left(G^{\prime}, U_{p}^{\prime}, \mathbb{Q}_{l}\right)$ by the images of the two degeneracy maps. Since spaces of functions on a finite set are naturally self-dual, we also have a $p$-new subspace $S_{2}\left(G^{\prime}, U_{p}^{\prime}, \mathbb{Q}_{l}\right)_{p \text {-new }}$ defined as the intersection of the kernels of the duals of the degeneracy maps.

Note that as finite sets, $X_{p}^{\prime}\left(\overline{\mathbb{F}}_{p}\right)$ and $X^{\prime}\left(\overline{\mathbb{F}}_{p}\right)$ are in bijection with the double coset spaces $G^{\prime}(\mathbb{Q}) \backslash G^{\prime}\left(\mathbb{A}_{\mathbb{Q}}\right) / U_{p}^{\prime}$ and $G^{\prime}(\mathbb{Q}) \backslash G^{\prime}\left(\mathbb{A}_{\mathbb{Q}}\right) / U^{\prime}$, respectively. These bijections identify the degeneracy maps on $S_{2}\left(G^{\prime}, U^{\prime}, \mathbb{Q}_{l}\right)$ with the pullback maps

$$
H_{\text {ét }}^{0}\left(\left(X^{\prime}\right)_{\overline{\mathbb{F}}_{p}}, \mathbb{Q}_{l}\right) \rightarrow H_{\text {ét }}^{0}\left(\left(X_{p}^{\prime}\right)_{\overline{\mathbb{F}}_{p}}, \mathbb{Q}_{l}\right)
$$


induced by the two degeneracy maps $X_{p}^{\prime} \rightarrow X^{\prime}$.

Theorem 7.3 The highest and lowest weight quotients of the weight filtration on $H^{d}\left(\left(X_{U, p}^{D}\right)_{\bar{K}}, \mathbb{Q}_{l}\right)$ are naturally isomorphic to $S_{2}\left(G^{\prime}, U_{p}^{\prime}, \mathbb{Q}_{l}\right)_{p \text {-new }}$ and $S_{2}\left(G^{\prime}, U_{p}^{\prime}, \mathbb{Q}_{l}\right)^{p \text {-new }}$, respectively.

Moreover, these isomorphisms are compatible with the actions of the primeto-p Hecke operators for $G$ and $G^{\prime}$, respectively.

Proof. We prove this statement for the lowest weight quotient; the corresponding statement for the top then follows by the fact that the weight spectral sequence is self-dual under Poincaré duality.

The bottom quotient of the weight filtration is the term $E_{2}^{d, 0}$ of the weight spectral sequence; that is, the cokernel of the map $E_{1}^{d-1,0} \rightarrow E_{1}^{d, 0}$. This is the restriction map:

$$
H_{\text {ét }}^{0}\left(Y^{(d-1)}, \mathbb{Q}_{l}\right) \rightarrow H_{\text {ét }}^{0}\left(Y^{(d)}, \mathbb{Q}_{l}\right) .
$$

By Proposition 6.1 the cokernel of this restriction map is naturally isomorphic to the cokernel of the map

$$
\bigoplus_{\tau} H_{\text {ét }}^{0}\left(\left(X_{\mathbb{S} \backslash\{\tau\}, \mathbb{S}}\right)_{\overline{\mathbb{F}}_{p}}, \mathbb{Q}_{l}\right) \oplus H_{\text {ét }}^{0}\left(\left(X_{\mathbb{S}, \mathbb{S} \backslash\{\tau\}}\right)_{\overline{\mathbb{F}}_{p}}, \mathbb{Q}_{l}\right) \rightarrow H_{\text {èt }}^{0}\left(\left(X_{\mathbb{S}, \mathbb{S}}\right)_{\mathbb{F}_{p}}, \mathbb{Q}_{l}\right) .
$$

For any given $\tau$, either $X_{\mathbb{S} \backslash\{\tau\}, \mathbb{S}}$ is contained in $X_{S, S^{\prime}}$ and $X_{\mathbb{S}, \mathbb{S} \backslash\{\tau\}}$ is contained in $X_{S^{\prime}, S}$, or vice versa.

Suppose $\tau \in S^{\prime}$, so $X_{\mathbb{S} \backslash\{\tau\}, \mathbb{S}}$ is contained in $X_{S, S^{\prime}}$. The scheme $X_{S, S^{\prime}}$ is a disjoint union of copies of $\left(\mathbb{P}^{1}\right)^{d}$, indexed by the points of $X^{\prime}$; the intersection of $X_{\mathbb{S} \backslash \tau, \mathbb{S}}$ with each of these copies of $\left(\mathbb{P}^{1}\right)^{d}$ is a rational curve. In particular pullback induces an isomorphism:

$$
H_{\text {ét }}^{0}\left(\left(X_{\mathbb{S} \backslash \tau, \mathbb{S}}\right)_{\overline{\mathbb{F}}_{p}}, \mathbb{Q}_{l}\right) \cong H_{\text {ét }}^{0}\left(\left(X_{S, S^{\prime}}\right)_{\overline{\mathbb{F}}_{p}}, \mathbb{Q}_{l}\right) \cong H_{\text {ét }}^{0}\left(X_{\overline{\mathbb{F}}_{p}}^{\prime}, \mathbb{Q}_{l}\right) .
$$

If we make these identifications, and we identify $X_{\mathbb{S}, \mathbb{S}}\left(\overline{\mathbb{F}}_{p}\right)$ with $X_{p}^{\prime}\left(\overline{\mathbb{F}}_{p}\right)$ via Proposition 7.1, the pullback map

$$
H_{\mathrm{ét}}^{0}\left(\left(X_{\mathbb{S} \backslash\{\tau\}, \mathbb{S}}\right)_{\overline{\mathbb{F}}_{p}}, \mathbb{Q}_{l}\right) \rightarrow H_{\mathrm{ét}}^{0}\left(\left(X_{\mathbb{S}, \mathbb{S}}\right)_{\overline{\mathbb{F}}_{p}}, \mathbb{Q}_{l}\right)
$$

becomes the degeneracy map

$$
H_{\text {ét }}^{0}\left(\left(X^{\prime}\right)_{\overline{\mathbb{F}}_{p}}, \mathbb{Q}_{l}\right) \rightarrow H_{\text {ét }}^{0}\left(\left(X_{p}^{\prime}\right)_{\overline{\mathbb{F}}_{p}}, \mathbb{Q}_{l}\right)
$$

that comes from "forgetting the level structure at p."

Similarly, the pullback map

$$
H_{\mathrm{ett}}^{0}\left(\left(X_{\mathbb{S}, \mathbb{S} \backslash\{\tau\}}\right)_{\overline{\mathbb{F}}_{p}}, \mathbb{Q}_{l}\right) \rightarrow H_{\text {ét }}^{0}\left(\left(X_{\mathbb{S}, \mathbb{S}}\right)_{\overline{\mathbb{F}}_{p}}, \mathbb{Q}_{l}\right)
$$

becomes the degeneracy map

$$
H_{\text {et }}^{0}\left(\left(X^{\prime}\right)_{\overline{\mathbb{F}}_{p}}, \mathbb{Q}_{l}\right) \rightarrow H_{\mathrm{et}}^{0}\left(\left(X_{p}^{\prime}\right)_{\overline{\mathbb{F}}_{p}}, \mathbb{Q}_{l}\right)
$$


that "mods out by the level structure at p."

If $\tau$ lies in $S$ rather than $S^{\prime}$, we still get the above two degeneracy maps, but in the opposite order.

Thus $E_{2}^{d, 0}$ is isomorphic to the quotient of $H_{\mathrm{et}}^{0}\left(\left(X_{p}^{\prime}\right)_{\overline{\mathbb{F}}_{p}}, \mathbb{Q}_{l}\right)$ by the image of the two degeneracy maps from $H_{\mathrm{et}}^{0}\left(X_{\mathbb{F}_{p}}^{\prime}, \mathbb{Q}_{l}\right)$. By the above discussion, this is isomorphic to $S_{2}\left(G^{\prime}, U_{p}^{\prime}, \mathbb{Q}_{l}\right)^{p \text {-new }}$.

For the Hecke equivariance, note that we have fixed an isomorphism between $G$ and $G^{\prime}$ at all finite places, and therefore can associate to every prime-to- $p$ Hecke operator for $G$ a corresponding prime-to- $p$ Hecke operator for $G^{\prime}$. Such Hecke operators act via correspondences on $X_{U, p}$ or $X_{p}^{\prime}$ defined in terms of the prime-to- $p$ level structures of the points of these varieties.

Since every construction in this paper is defined in terms of $p$-isogenies, these constructions commute with correspondences defined in this way, and are thus compatible with the Hecke actions in the above sense.

Remark 7.4 The above construction can be easily adapted to work with coefficients in an arbitrary lisse sheaf over $\overline{\mathbb{Q}}_{l}$. Thus the result above extends to "higher weight". In certain circumstances one can even obtain results with coefficients in $\mathbb{Z}_{l}$; the main difficulty is that the weight spectral sequence is not known to degenerate at $E_{2}$ in this case. This can be checked by hand in many cases, such as when $d=2$.

A special case of Jacquet-Langlands for $G$ and $G^{\prime}$ follows:

Corollary 7.5 Under the assumptions of this section, let $\pi$ be an automorphic representation of $G$ (resp. $G^{\prime}$ ) that is cohomological, and Steinberg at a prime $p$ split in $E$ and inert in $F^{+}$. Then there is an automorphic representation $\pi^{\prime}$ of $G^{\prime}$ (resp. $G$ ) isomorphic to $\pi$ at all non-archimedean places.

\section{Appendix: Bilinear pairings}

Let $E, F^{+}$, and $F$ be as in section 2, and let $D$ be a central simple $F$-algebra of dimension $n^{2}$, equipped with an involution $\alpha \mapsto \bar{\alpha}$ of the second kind. Let $\mathcal{V}$ be a free left $D$-module of rank one, equipped with a pairing $\langle\rangle:, V \times V \rightarrow \mathbb{Q}$ that is alternating and nondegenerate, and satisfies $\langle\alpha x, y\rangle=\langle x, \bar{\alpha} y\rangle$.

Note that $D$ is split at infinity, as $F$ is purely imaginary. For each real embedding $\tau$ of $F^{+}, D \otimes_{F^{+}, \tau} \mathbb{R}$ is isomorphic to $M_{n}(\mathbb{C})$. For a suitable choice of idempotent $e$ in $D \otimes_{F^{+}, \tau} \mathbb{R}$ such that $e=\bar{e}, e\left(\mathcal{V} \otimes_{F^{+}, \tau} \mathbb{R}\right)$ is an $n$-dimensional complex vector space, on which $\langle$,$\rangle induces a nondegenerate Hermitian pairing.$ We let $r_{\tau}(\mathcal{V})$ and $s_{\tau}(\mathcal{V})$ denote the number of 1's and -1 's in the signature of this pairing; this is independent of the particular idempotent chosen and satisfies $r_{\tau}(\mathcal{V})+s_{\tau}(\mathcal{V})=n$.

We are concerned with the question of determining whether, for given $r_{\tau}^{\prime}$ and $s_{\tau}^{\prime}$, there exists a $\mathcal{V}^{\prime}$ and $\langle,\rangle^{\prime}$ such that the pair $\left(\mathcal{V}^{\prime},\langle,\rangle^{\prime}\right)$ is isomorphic to $(\mathcal{V},\langle\rangle$,$) over \mathbb{A}_{\mathbb{Q}}^{f}$ but such that $r_{\tau}\left(\mathcal{V}^{\prime}\right)=r_{\tau}^{\prime}$ and $s_{\tau}\left(\mathcal{V}^{\prime}\right)=s_{\tau}^{\prime}$. This turns out to be an easy application of results of Kottwitz [Ko]. 
Let $G$ be the subgroup of $\operatorname{Aut}_{D}(\mathcal{V})$ of automorphisms preserving $\langle$,$\rangle . (Note$ that this is different from the $G$ appearing in the main body of the text, as we are not working up to a scalar multiple.) The cohomology set $H^{1}(\mathbb{Q}, G)$ is in bijection with the set of left $D$-modules $\mathcal{V}^{\prime}$ of rank one with pairings $\langle,\rangle^{\prime}$ of the sort under consideration. A pair $\left(\mathcal{V}^{\prime},\langle,\rangle^{\prime}\right)$ is isomorphic to $(\mathcal{V},\langle\rangle$,$) over \mathbb{A}_{\mathbb{Q}}^{f}$ if and only if the corresponding class in $H^{1}\left(Q_{v}, G\right)$ is trivial for all finite places $v$ of $\mathbb{Q}$.

It thus suffices to understand the image of the natural map

$$
H^{1}(Q, G) \rightarrow \oplus_{v} H^{1}\left(Q_{v}, G\right)
$$

where $v$ runs over the places of $\mathbb{Q}$. Let $A(G)$ denote $\pi_{0}\left(Z(\hat{G})^{\operatorname{Gal}(\overline{\mathbb{Q}} / \mathbb{Q})}\right)^{\vee}$, i.e., the Pontryagin dual of group of components of the Galois invariants of the center of the connected Langlands dual of $G$. Similarly let $A\left(G_{v}\right)$ denote $\pi_{0}\left(Z\left(\hat{G}_{v}\right)^{\operatorname{Gal}\left(\overline{\mathbb{Q}_{v}} / \mathbb{Q}_{v}\right)}\right)^{\vee}$. Then Kottwitz [Ko] constructs for each $v$ a natural map

$$
H^{1}\left(Q_{v}, G\right) \rightarrow A\left(G_{v}\right),
$$

and shows ([Ko], Proposition 2.6) that the image of $H^{1}(Q, G)$ in $\oplus_{v} H^{1}\left(Q_{v}, G\right)$ is equal to the kernel of the composite map

$$
\oplus_{v} H^{1}\left(Q_{v}, G\right) \rightarrow \oplus_{v} A\left(G_{v}\right) \rightarrow A(G) .
$$

In light of this, understanding our question amounts to understanding the map $A\left(G_{\infty}\right) \rightarrow A(G)$. The group $G \times_{\mathbb{Q}} \mathbb{R}$ is isomorphic to a product

$$
\prod_{\tau \in \mathbb{S}} U\left(r_{\tau}, s_{\tau}\right)
$$

of unitary groups.

Over $\mathbb{C}$, a unitary group $U(r, s)$ becomes isomorphic to $\mathrm{GL}_{n}(\mathbb{C})$, and comes equipped with a natural action of $\operatorname{Gal}(\mathbb{C} / \mathbb{R})$. Complex conjugation acts on this $\mathrm{GL}_{n}(\mathbb{C})$ by sending a matrix $g$ to $\left(g^{*}\right)^{-1}$, where $*$ denotes adjoint with respect to a Hermitian pairing on $\mathbb{C}^{n}$ with signature consisting of $r$ 1's and $s-1$ 's. If we fix a maximal torus of $T$ of $U(r, s)$, the induced action on the character group of $T$ sends a character to its inverse.

This action of $\operatorname{Gal}(\mathbb{C} / \mathbb{R})$ on the character group of $T$ induces an action of $\operatorname{Gal}(\mathbb{C} / \mathbb{R})$ on the root datum of $\mathrm{GL}_{n}(\mathbb{C})$, and hence also on the dual root datum. This action in turn defines a unique action of $\operatorname{Gal}(\mathbb{C} / \mathbb{R})$ on the Langlands dual of $\mathrm{GL}_{n}(\mathbb{C})$ that respects the complex structure of $\mathrm{GL}_{n}(\mathbb{C})$. (Note that by contrast the Galois action on $U(r, s) \times_{\mathbb{R}} \mathbb{C}$ did not respect this complex structure.) Thus the connected Langlands dual of $U(r, s)$ is again $\mathrm{GL}_{n}(\mathbb{C})$, equipped with an action of $\operatorname{Gal}(\mathbb{C} / \mathbb{R})$. The action of complex conjugation on the maximal torus of this group can be read off of the action on the root datum; it sends any element of the maximal torus to its inverse. In particular, $Z(\widehat{U}(r, s))^{\mathrm{Gal}(\mathbb{C} / \mathbb{R})}$ is equal to

$$
\left\{x \in \mathbb{C}: x=x^{-1}\right\}=\{ \pm 1\} .
$$


Moreover, under the correspondence between $H^{1}(\mathbb{R}, U(r, s))$ and isomorphism classes of nondegenerate Hermitian forms on $\mathbb{C}^{r+s}$, the element of $H^{1}(\mathbb{R}, U(r, s))$ corresponding to a Hermitian form of signature consisting of $r^{\prime} 1$ 's and $s^{\prime}-1$ 's maps to the element $(-1)^{r-r^{\prime}}$ under the map $H^{1}(\mathbb{R}, U(r, s)) \rightarrow A(U(r, s))$.

Thus we have a natural isomorphism:

$$
A\left(G_{\infty}\right) \rightarrow \prod_{\tau \in \mathbb{S}}\{ \pm 1\} .
$$

On the other hand, we can compute $A(G)$ in exactly the same fashion. In particular $G \times_{\mathbb{Q}} E$ is isomorphic to $D^{\times}$, and $\operatorname{Gal}(E / \mathbb{Q})$ acts on $B^{\times}$by sending $\alpha$ to $\bar{\alpha}^{-1}$. Thus the center of $G \times_{\mathbb{Q}} \overline{\mathbb{Q}}$ is isomorphic to $\left(F \otimes_{E} \overline{\mathbb{Q}}\right)^{\times}$, where $\operatorname{Gal}(\overline{\mathbb{Q}} / \mathbb{Q})$ acts on $F$ via the action of $\operatorname{Gal}(E / \mathbb{Q})$ on $B$ and on $\overline{\mathbb{Q}}$ in the obvious way.

If we fix a maximal torus $T$ of $G$, we obtain a sequence of maps

$$
\chi(Z(\hat{G}))^{*} \rightarrow \chi(\hat{T})^{*}=\chi(T) \rightarrow \chi(Z(G))
$$

where $\chi$ denotes the character group. This identifies $\chi(Z(\hat{G}))^{*}$ with a sublattice of $\chi(Z(G))$ in a manner that respects the action of $\operatorname{Gal}(\overline{\mathbb{Q}} / \mathbb{Q})$. Moreover, because over $\overline{\mathbb{Q}}, G$ is simply a product of copies of $\mathrm{GL}_{n}$, the image of this sequence of maps is just $n \chi(Z(G))$. It follows that $Z(\hat{G})$ is simply $\left(\mathbb{C}^{\times}\right)^{\mathbb{S}}$, on which $\operatorname{Gal}\left(F^{+} / \mathbb{Q}\right)$ acts by permuting the factors and complex conjugation in $\operatorname{Gal}(E / \mathbb{Q})$ acts by inversion. In particular $Z(\hat{G})^{\operatorname{Gal}(\overline{\mathbb{Q}} / \mathbb{Q})}$ is simply \pm 1 . The map $A\left(G_{\infty}\right) \rightarrow A(G)$ simply takes an element of $\prod_{\tau \in \mathbb{S}}\{ \pm 1\}$ and multiplies the entries together.

The upshot of this is:

Proposition 8.1 Let $r_{\tau}^{\prime}$ and $s_{\tau}^{\prime}$ be integers between 0 and $n$ such that $s_{\tau}^{\prime}=$ $n-r_{\tau}^{\prime}$ for all $\tau$. Then there exists a $\left(\mathcal{V}^{\prime},\langle,\rangle^{\prime}\right)$ isomorphic to $(\mathcal{V},\langle\rangle$,$) at all finite$ places but with archimedean invariants $r_{\tau}^{\prime}, s_{\tau}^{\prime}$ if and only if

$$
\sum_{\tau} r_{\tau} \equiv \sum_{\tau} r_{\tau}^{\prime} \bmod 2
$$

Proof. Let $x$ be the class in $\oplus_{v} H^{1}\left(\mathbb{Q}_{v}, G\right)$ that is trivial at all finite places but that corresponds to $r_{\tau}^{\prime}, s_{\tau}^{\prime}$ at infinity. Then $x$ maps to $(-1)^{\sum_{\tau} r_{\tau}-r_{\tau}^{\prime}}$ in $A(G)$, and is thus in the kernel of the map to $A(G)$ if and only if the parity condition given above is satisifed. Since the kernel of this map is equal to the image of $H^{1}(Q, G)$, the result follows immediately.

Corollary 8.2 Let $\mathcal{V}$ be an $n$-dimensional $F$-vector space, together with an althernating nondegenerate pairing $\langle\rangle:, \mathcal{V} \times \mathcal{V} \rightarrow \mathbb{Q}$, such that $\langle\alpha x, y\rangle=\langle x, \bar{\alpha} y\rangle$. Let $r_{\tau}^{\prime}$ and $s_{\tau}^{\prime}$ be integers between 0 and $n$ such that $s_{\tau}^{\prime}=n-r_{\tau}^{\prime}$ for all $\tau$. Then there exists a $\left(\mathcal{V}^{\prime},\langle,\rangle^{\prime}\right)$ isomorhpic to $(\mathcal{V},\langle\rangle$,$) at all finite places but with$ archimedean invariants $r_{\tau}^{\prime}, s_{\tau}^{\prime}$ if and only if

$$
\sum_{\tau} r_{\tau} \equiv \sum_{\tau} r_{\tau}^{\prime} \bmod 2
$$


Proof. Let $D=\operatorname{GL}_{n}(F)$, and let $\alpha \mapsto \bar{\alpha}$ be the conjugate transpose on $D$. Then to any $(\mathcal{V},\langle\rangle$,$) as in the statement of the corollary, we can associate the$ left $D$-module $\mathcal{V}^{n}$, together with the natural pairing induced by $\langle$,$\rangle . This is an$ equivalence of categories between $(\mathcal{V},\langle\rangle$,$) as in the statement of the corollary$ and those considered in the preceding proposition (for $D=G L_{n}(F)$ ). Thus the corollary follows immediately from the preceding proposition.

Acknowledgements. The author is grateful to Richard Taylor for his continued interest and helpful suggestions, to Johan de Jong for his help with the deformation theory of abelian varieties, to Brian Osserman for many helpful conversations, and to Marc Hubert-Nicole for his helpful comments on an earlier version of this paper. The author was partially supported by the National Science Foundation.

\section{References}

[dJ] de Jong, A. J The moduli spaces of principally polarized abelian varieties with $\Gamma_{0}(p)$-level structure. J. Algebraic Geom. 2 (1993), no. 4, 667-688.

[Fu] Fulton, R. Introduction to Toric Varieties, Princeton University Press (1993).

[Gh1] Ghitza, A. Hecke eigenvalues of Siegel modular forms ( $\bmod p)$ and of algebraic modular forms. J. Number Theory 106 (2004), no. 2, 345-384.

[Gh2] Ghitza, A. All Siegel Hecke eigensystems (mod $p)$ are cuspidal. Math. Res. Lett. 13 (2006), no. 5-6, 813-823.

[Go] Goren, E. Hasse invariants for Hilbert modular varieties. Israel J. Math. 122 (2001), 157-174.

[GO] Goren, E., Oort, F. Stratifications of Hilbert modular varieties. J. Algebraic Geom. 9 (2000), no. 1, 111-154.

[Gr] Grothendieck, A. Groupes de Barsotti-Tate et cristaux de Dieudonné. Séminaire de Mathématiques Supérior 45, Presses de l'Université de Montréal (1974).

[Gross] Gross, B. Algebraic modular forms. Israel J. Math. 113 (1999), 61-93.

[Ha] Hartshorne, R. Algebraic Geometry. Springer-Verlag (1977).

[JL] Jacquet, H., Langlands, R. P. Automorphic forms on GL(2). Lecture Notes in Math. Vol. 114, Springer-Verlag (1970).

[Ko] Kottwitz, R. Stable trace formula: elliptic singular terms Math. Ann. 275 (1986), no. 3, 365-399.

[MM] Mazur, B., Messing, W. Universal extensions and one-dimensional crystalline cohomology. Lecture Notes in Math. Vol. 370, Springer-Verlag (1974). 
[Mo] Moonen, B. A dimension formula for Ekedahl-Oort strata, Ann. Inst. Fourier (Grenoble) 54 (2004), no. 3, 666-698.

[Oo] Oort, F. A stratification of a moduli space of Abelian varieties, in Moduli of Abelian Varieties. (Texel, 1999), Progr. Math. 195, Birkhauser (2001), 345416.

[Pa] Pappas, G. Arithmetic models for Hilbert-Blumenthal modular varieties. $\mathrm{PhD}$ thesis, Columbia University, unpublished (1992).

[Ri1] Ribet, K. On modular representations of $\mathrm{Gal}(\overline{\mathbb{Q}} / \mathbb{Q})$ arising from modular forms Invent. Math. 100 (1990), no. 2, 431-476.

[Ri2] Ribet, K. Bimodules and abelian surfaces. In Algebraic Number Theory, Adv. Stud. Pure Math. 17, Academic Press (1989), 359-407.

[RZ1] Rapoport, M., Zink, T. Period Spaces for p-divisible Groups Annals of Math. Studies 141, Princeton University Press (1996).

[RZ2] Rapoport, M., Zink, T. Über die lokale Zetafunktion von Shimuravarietäten, Monodromiefiltrations und verschwindende Zyklen in ungleicher Characteristik, Inv. Math. 68 (1980), 21-101.

[Sa] Saito, T., Weight spectral Sequences and independence of $l$ J. Inst. Math. Jussieu 2 (2003), no. 4, 583-634.

[Se] Serre, J. P. Two letters on quaternions and modular forms (mod $p$ ) Israel J. Math. 95 (1996) 281-299.

[St] Stamm, H. On the reduction of the Hilbert-Blumenthal moduli scheme with $\Gamma_{0}(p)$ level structure, Forum Math. 9 (1997), no. 4, 405-455.

[We] Wedhorn, T. The dimension of Oort strata of Shimura varieties of PEL type, in Moduli of Abelian Varieties (Texel, 1999), Progr. Math. 195, Birkhauser (2001), 441-471.

[Zi] Zink, T. Über die schlechte Reduktion einiger Shimuramannigfaltigkeiten. Compositio Math. 45 (1982), no. 1, 15-107. 